\title{
Stellar Pulsation and the Production of Dust and Molecules in Galactic Carbon Stars
}

\section{DOI:}

10.3847/1538-4357/ab4f6b

\section{Document Version}

Final published version

Link to publication record in Manchester Research Explorer

\section{Citation for published version (APA):}

Kraemer, K. E., Sloan, G. C., Keller, L. D., Mcdonald, I., Zijlstra, A., \& Groenewegen, M. A. T. (2019). Stellar Pulsation and the Production of Dust and Molecules in Galactic Carbon Stars. The Astrophysical Journal, 887(1). https://doi.org/10.3847/1538-4357/ab4f6b

\section{Published in:}

The Astrophysical Journal

\section{Citing this paper}

Please note that where the full-text provided on Manchester Research Explorer is the Author Accepted Manuscript or Proof version this may differ from the final Published version. If citing, it is advised that you check and use the publisher's definitive version.

\section{General rights}

Copyright and moral rights for the publications made accessible in the Research Explorer are retained by the authors and/or other copyright owners and it is a condition of accessing publications that users recognise and abide by the legal requirements associated with these rights.

\section{Takedown policy}

If you believe that this document breaches copyright please refer to the University of Manchester's Takedown Procedures [http://man.ac.uk/04Y6Bo] or contact uml.scholarlycommunications@manchester.ac.uk providing relevant details, so we can investigate your claim.

\section{OPEN ACCESS}




\title{
Stellar Pulsation and the Production of Dust and Molecules in Galactic Carbon Stars
}

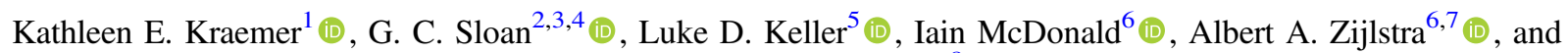 \\ Martin A. T. Groenewegen ${ }^{8}$ (i) \\ ${ }^{1}$ Institute for Scientific Research, Boston College, 140 Commonwealth Avenue, Chestnut Hill, MA 02467, USA; kathleen.kraemer@bc.edu \\ ${ }^{2}$ Space Telescope Science Institute, 3700 San Martin Drive, Baltimore, MD 21218, USA; sloan@astro.cornell.edu \\ ${ }^{3}$ Department of Physics and Astronomy, University of North Carolina, Chapel Hill, NC 27599-3255, USA \\ ${ }^{4}$ Center for Astrophysics and Planetary Science, Cornell University, Ithaca, NY 14853-6801, USA \\ ${ }^{5}$ Department of Physics and Astronomy, Ithaca College, Ithaca, NY 14850, USA \\ ${ }^{6}$ Jodrell Bank Centre for Astrophysics, Alan Turing Building, University of Manchester, Manchester, M13 9PL, UK \\ ${ }^{7}$ Laboratory for Space Research, The University of Hong Kong, Pokfulam Road, Hong Kong \\ ${ }^{8}$ Koninklijke Sterrenwacht van België, Ringlaan 3, B-1180 Brussels, Belgium \\ Received 2019 September 19; revised 2019 October 16; accepted 2019 October 17; published 2019 December 12
}

\begin{abstract}
New infrared spectra of 33 Galactic carbon stars from FORCAST on SOFIA reveal strong connections between stellar pulsations and the dust and molecular chemistry in their circumstellar shells. A sharp boundary in overall dust content, which predominantly measures the amount of amorphous carbon, separates the semiregular and Mira variables, with the semiregulars showing little dust in their spectra and the Miras showing more. In semiregulars, the contribution from $\mathrm{SiC}$ dust increases rapidly as the overall dust content grows, but in Miras, the SiC dust feature grows weaker as more dust is added. A similar dichotomy is found with the absorption band from CS at $\sim 7.3 \mu \mathrm{m}$, which is generally limited to semiregular variables. Observationally, these differences make it straightforward to distinguish semiregular and Mira variables spectroscopically without the need for long-term photometric observations or knowledge of their distances. The rapid onset of strong SiC emission in Galactic carbon stars in semiregular variables points to a different dust-condensation process before strong pulsations take over. The break in the production of amorphous carbon between semiregulars and Miras seen in the Galactic sample is also evident in Magellanic carbon stars, linking strong pulsations in carbon stars to the strong mass-loss rates which will end their lives as stars across a wide range of metallicities.
\end{abstract}

Unified Astronomy Thesaurus concepts: Carbon stars (199); Circumstellar matter (241); Long period variable stars (935); Spectroscopy (1558)

Supporting material: machine-readable tables

\section{Introduction}

A fundamental problem in astrophysics is understanding how stars in the late stages of their evolution enrich galaxies with dust and freshly fused elements. It is currently uncertain what the relative contributions are from the most massive stars, which will explode as supernovae, creating new elements but likely destroying dust, compared to lower-mass stars (e.g., Micelotta et al. 2018; Dell'Agli et al. 2019; Nanni et al. 2019). Low- and intermediate-mass stars create dust grains in the cool outer layers of their atmospheres, and the radiation pressure on these grains then helps drive the mass-loss process (e.g., Groenewegen \& Sloan 2018; Höfner \& Olofsson 2018, and references therein). Dust serves as a readily observable tracer of the total mass loss, and it is easily measured in the outflows from evolved stars. Infrared surveys of the Large and Small Magellanic Clouds (LMC and SMC) have pointed to carbon stars as the dominant source of dust being pumped into the interstellar medium (ISM) by stars (e.g., Matsuura et al. 2009; Boyer et al. 2011).

A significant fraction of intermediate-mass stars may end their lives on the asymptotic giant branch (AGB) as carbon stars, due to the production of carbon in their interiors and its dredge-up to their surfaces. The AGB stars become carbon stars when their $\mathrm{C} / \mathrm{O}$ ratios exceed unity. In these stars, free carbon remains after the formation of $\mathrm{CO}$, leading to a carbonrich, rather than oxygen-rich, gas and dust chemistry (e.g., Habing 1996; Wallerstein \& Knapp 1998, and references therein). The Infrared Spectrograph (IRS; Houck et al. 2004) on the Spitzer Space Telescope (Werner et al. 2004) has been used to study carbon stars in the low-metallicity environments of the LMC (e.g., Matsuura et al. 2006; Zijlstra et al. 2006; Leisenring et al. 2008), the SMC (e.g., Sloan et al. 2006; Lagadec et al. 2007), and other Local Group galaxies (e.g., Matsuura et al. 2007; Sloan et al. 2012).

The key to those studies, particularly in the Magellanic Clouds, has been samples of sufficient size. The combined IRS programs used by Sloan et al. (2016) had 144 carbon stars in the LMC and 40 in the SMC. Their control sample for the much larger Milky Way, however, contained only 42 carbon stars. The Galactic sample was observed with the ShortWavelength Spectrometer (SWS; de Graauw et al. 1996) on the Infrared Space Observatory (ISO; Kessler et al. 1996) and served as a valuable comparison sample for many of the studies of extragalactic carbon stars mentioned above. However, it is a selective and relatively small sample given the size of the Milky Way. While larger samples of infrared spectra were obtained with the low-resolution spectrometer (LRS; Olnon et al. 1986) on the Infrared Astronomical Satellite (IRAS; Neugebauer et al. 1984), the shortest wavelength is $7.67 \mu \mathrm{m}$, too red to measure important spectral diagnostics.

To improve the Galactic sample, we obtained 4.9-13.7 $\mu \mathrm{m}$ spectra of Galactic carbon stars using the Faint Object infraRed CAmera for the SOFIA Telescope (FORCAST; Herter et al. 2012) on the Stratospheric Observatory for Infrared Astronomy (SOFIA; Young et al. 2012). Section 2 describes our source 
selection, observations, and data processing. The results are given in Section 3 and discussed in Section 4, including comparisons to the SWS sample and the Magellanic Cloud samples. Section 5 summarizes our findings. The Appendix details the changes to the procedures for extracting spectral features needed to avoid telluric contamination.

\section{Source Selection and Observations}

\subsection{Source Selection}

The Galactic sample from the SWS consists of observations from numerous individual projects and suffers from inevitable biases due to the various selection criteria used. In particular, the SWS sample has a deficit of semiregular variables, which pulsate with weaker amplitudes than Miras. The SWS sample also selected against carbon stars with longer pulsation periods, especially $P>400$ days, compared to the Magellanic samples (Figure 1, top two panels). These sources are the most embedded objects with the most optically thick dust shells, and as noted above, they may dominate the dust returned to the ISM by stars.

To address the biases in the SWS data, we started with samples of Galactic carbon stars from Sloan et al. (1998), Jones et al. (1990), Nakashima et al. (2000), and Whitelock et al. (2006). Using their periods and the IRAS photometry, we selected sources in three groups. Group 1 has $\mathrm{SRb}$ variables with $F_{\nu}(12 \mu \mathrm{m})>40 \mathrm{Jy}$; Group 2 contains Mira variables with 400 days $<P<500$ days and $F_{\nu}>60 \mathrm{Jy}$; and Group 3 consists of Miras with $P>550$ days and $F_{\nu}>150 \mathrm{Jy} .{ }^{9}$ Our sample originally had 22 stars in Group 1, 8 in Group 2, and 13 in Group 3. Due to scheduling constraints that necessitated using back-up targets, as well as the nature of SOFIA Survey Programs, the observed sample consists of 11 Group 1 stars, 6 Group 2 stars, and 18 Group 3 stars, which yielded 33 usable spectra. Table 1 provides details on the observed stars.

\subsection{FORCAST Observations and Pipeline Processing}

SOFIA observed the stars in service mode over the course of three cycles, Cycle 1 (2013, 4 stars, Plan ID =01_0041), Cycle 3 (2015, 10 stars, 03_0104), and Cycle 4 (2016, 21 stars, 04_0129). We used the two low-resolution grism settings in FORCAST, G063 and G111, to obtain spectra over the wavelength ranges $4.9-7.8$ and $8.3-13.8 \mu \mathrm{m}$, respectively (Keller et al. 2010). The observations were made with the 4."7 slit, which results in a spectral resolution of $R \sim 120$. The 31 stars from Cycles 3 and 4 were processed by the SOFIA Data Cycle System with pipeline version 1_2_0. Those from Cycle 1 were processed with pipeline version 1_0_0; they could not be reprocessed due to changes in the observing configuration after Cycle 1. Two sources (CIT 6 and T Cnc) were discarded as their data were inconsistent both with previous observations and between the G063 and G111 spectra. Thus, our final sample consists of 33 stars. The bottom two panels in Figure 1 show our new SOFIA sample and the combined sample for the Milky Way.

\subsection{Comparison Samples}

The comparison sample for the Milky Way comes from the SWS archive of Sloan et al. (2003b), and carbon-rich sources

\footnotetext{
9 The flux constraints were driven by the desire for short integration times and a reasonable sample size.
}

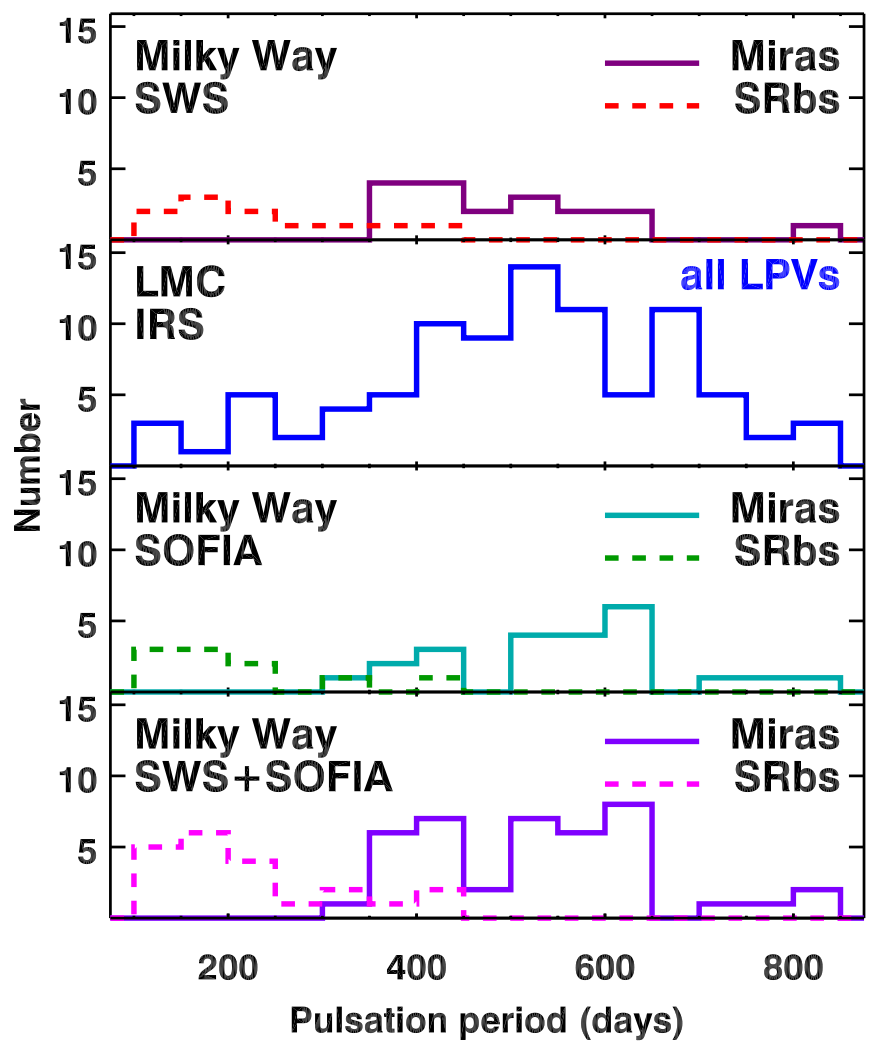

Figure 1. Galactic and LMC carbon stars with 5-14 $\mu \mathrm{m}$ spectra. (Top) Galactic stars with SWS spectra; (second from top) LMC long period variables (LPVs) with IRS spectra; (second from bottom) Galactic stars with new SOFIA spectra; (bottom) combined SWS and SOFIA sample of Galactic stars. The Galactic samples are separated into Miras (solid lines) and SRbs (dashed lines).

were selected using the spectral classifications by Kraemer et al. (2002). We used the variability types and periods from the General Catalog of Variable Stars (GCVS; Samus et al. 2017) to assign 31 of the 42 sources to one of the three SOFIAselection groups if possible ${ }^{10}$; two Miras with periods of 358 and 389 days were placed in Group 2. Of the 11 SWS sources that did not fit in these groups, 3 are SRa variables (2 of which have known periods), 4 were Lb variables (i.e., irregulars), and 4 have no variability type assigned in the GCVS.

We use the Magellanic Cloud sample from Sloan et al. (2016), who compiled and uniformly processed IRS observations of carbon stars from 11 Spitzer programs. Although the Magellanic sample does come from several separate programs, some of those projects were large surveys that attempted to sample a broad range of sources. Thus, the Magellanic sample, though not completely free of selection biases, is nonetheless less biased than the SWS sample.

As with the Galactic samples, we assign the Magellanic sources to one of the three groups, using variability types and periods from Soszyński et al. $(2009,2011)$ for the LMC and SMC, respectively. These are based on data from the Optical Gravitational Lensing Experiment (OGLE; Udalski et al. 1992). For the LMC, 55 of the 144 sources could be assigned a group, and for the SMC 32 of 40. We have left Magellanic Miras with periods below 395 days unassigned for better comparison with the Galactic sample. OGLE does not

\footnotetext{
${ }^{10}$ We dropped the flux criterion (Section 2.1). Also, periods for V Aql and Y CVn have been updated since Sloan et al. (2016) to 400 and 268 days, respectively.
} 
Table 1

FORCAST Targets

\begin{tabular}{|c|c|c|c|c|c|c|c|}
\hline $\begin{array}{l}\text { Star } \\
\text { Name }\end{array}$ & R.A. & Decl. & $\begin{array}{c}\text { Obs. Date } \\
\text { (yyyy mmm dd) }\end{array}$ & $\begin{array}{l}\text { Period } \\
\text { (days) }\end{array}$ & $\begin{array}{l}F_{12} \\
(\mathrm{Jy})\end{array}$ & Group & $\begin{array}{l}\text { Period } \\
\text { Ref. }\end{array}$ \\
\hline WZ Cas & 000115.86 & +602119.0 & 2016 Sep 21 & 186 & 44 & 1 & S98 \\
\hline ST Cam & $0451 \quad 13.35$ & +681007.6 & 2013 Sep 13 & 300 & 95 & 1 & S98 \\
\hline Y Tau & 054539.41 & +204142.2 & 2016 Sep 22 & 242 & 144 & 1 & S98 \\
\hline TU Gem & 061053.10 & +260053.4 & 2015 Nov 10 & 230 & 70 & 1 & S98 \\
\hline UU Aur & 063632.84 & +382643.8 & 2013 Sep 13 & 234 & 232 & 1 & S98 \\
\hline $\mathrm{X} \mathrm{Cnc}$ & 085522.88 & +171352.6 & 2015 May 30 & 195 & 90 & 1 & S98 \\
\hline $\mathrm{T} \mathrm{Cnc}^{\mathrm{a}}$ & 085640.15 & +195057.0 & 2015 Jun 5 & 482 & 61 & 1 & S98 \\
\hline U Нya & 103733.27 & -132304.3 & 2015 Jun 3 & 450 & 206 & 1 & S98 \\
\hline TW Oph & 172943.66 & -192822.9 & 2016 Feb 18 & 185 & 96 & 1 & S98 \\
\hline RT Cap & 201706.53 & -211904.5 & 2016 Jul 14 & 393 & 73 & 1 & S98 \\
\hline RV Cyg & 214316.33 & +380103.0 & 2015 May 30 & 263 & 103 & 1 & S98 \\
\hline KY Cam & 032759.03 & +604455.2 & 2016 Sep 27 & 477: & 70 & 2 & N00 \\
\hline V718 Tau & 043121.93 & +173910.3 & 2015 Sep 18 & 405 & 72 & 2 & S98 \\
\hline R Lep & 045936.35 & -144822.5 & $2016 \mathrm{Feb} 10$ & 427 & 380 & 2 & S98 \\
\hline CL Mon & 065536.69 & +062243.2 & 2015 Nov 6 & 497 & 113 & 2 & S98 \\
\hline U Cyg & 201936.59 & +475339.1 & 2015 Jun 4 & 463 & 112 & 2 & S98 \\
\hline AX Cep & 212654.03 & +701315.4 & 2016 Sep 21 & 395 & 72 & 2 & S98 \\
\hline V668 Cas & 002741.13 & +693851.6 & 2016 Sep 20 & 650 & 306 & 3 & J90 \\
\hline V370 Aur & 054349.69 & +324206.2 & 2016 Feb 5 & 780 & 196 & 3 & J90 \\
\hline V1259 Ori & 060400.05 & +072552.0 & 2016 Feb 5 & 686 & 320 & 3 & J90 \\
\hline CGCS 6276 & 080920.26 & -362426.8 & 2016 Feb 5 & 832 & 156 & 3 & W06 \\
\hline V346 Pup & 081048.89 & -325205.6 & 2016 Feb 5 & 568 & 347 & 3 & W06 \\
\hline CQ Pyx & 091353.94 & -245125.2 & 2016 Feb 5 & 659 & 737 & 3 & W06 \\
\hline IRC +10216 & 094757.41 & +131643.6 & 2015 Nov 20 & 651 & 47530 & 3 & W06 \\
\hline CGCS 2653 & 095306.72 & -533853.5 & 2016 Jul 12 & 630 & 157 & 3 & W06 \\
\hline $\operatorname{CIT} 6^{\mathrm{a}}$ & 101602.28 & +303419.0 & 2013 Oct 25 & 617 & 3319 & 3 & W06 \\
\hline CGCS 2987 & 111638.90 & -655056.0 & 2016 Jul 18 & 623 & 181 & 3 & W06 \\
\hline V1132 Cen & 124209.58 & -435502.9 & 2016 Jul 18 & 551 & 159 & 3 & W06 \\
\hline CGCS 3311 & 125715.79 & -690151.5 & 2016 Jul 14 & 586 & 264 & 3 & W06 \\
\hline V2548 Oph & 170758.11 & -244431.2 & 2016 Feb 18 & 747 & 793 & 3 & W06 \\
\hline RAFGL 2154 & 182639.48 & -065403.6 & 2016 Feb 11 & 635 & 220 & 3 & W06 \\
\hline CGCS 4014 & 182734.22 & -08 3723.4 & 2016 Sep 21 & 659 & 153 & 3 & W06 \\
\hline V1420 Aq1 & 192018.12 & $\begin{array}{lll}-08 & 02 & 12.1\end{array}$ & 2013 Sep 19 & 694 & 384 & 3 & W06 \\
\hline V1969 Cyg & 200914.24 & +312544.9 & 2015 Nov 20 & 550 & 173 & 3 & J90 \\
\hline V384 Cep & 222553.47 & +602043.6 & 2016 Sep 21 & 698: & 182 & 3 & NOO \\
\hline
\end{tabular}

Note.

a Observations problematic and not included in analysis.

References. J90: Jones et al. (1990); S98: Sloan et al. (1998), Kholopov et al. (1992); N00: Nakashima et al. (2000); W06: Whitelock et al. (2006).

distinguish among different semiregular categories. We have treated them as SRa or SRb depending on their position in period-luminosity space (see Section 4.4): 4 of the 13 SRs in the LMC sample are SRbs and 3 of the 12 SMC SRs are SRbs.

\section{Results and Analysis}

\subsection{The Spectra}

The new FORCAST spectra for most of the Galactic carbon stars are dominated by the $\mathrm{SiC}$ dust emission feature at $\sim 11 \mu \mathrm{m}$ in the G111 spectral range, while the G063 data show absorption features from carbonaceous molecules such as $\mathrm{CO}$, $\mathrm{C}_{2} \mathrm{H}_{2}$, and CS. Figures 2-4 show the data for Groups 1-3, respectively. The wavelength range from 9.25 and $10.1 \mu \mathrm{m}$, shown in gray, is contaminated by telluric ozone that was incompletely removed due to changes in the atmospheric absorption between observations of science and calibration targets. The range from 7.6 to $7.7 \mu \mathrm{m}$ can also show telluric contamination in some of the spectra (e.g., ST Cam). An instrumental artifact is present in some spectra, causing a spike below $\sim 5.2 \mu \mathrm{m}$ and a drop above $13.5 \mu \mathrm{m} .{ }^{11}$ These data are excluded from further analysis, so the $\mathrm{C}_{2} \mathrm{H}_{2}$ absorption feature at $13.7 \mu \mathrm{m}$ was not considered.

\subsection{Spectral Feature Extraction}

We use the Manchester system (Sloan et al. 2006; Zijlstra et al. 2006) to measure the [6]-[9] color and the strength of the silicon carbide dust feature (SiC) relative to the local continuum (Figure 5). The [6]-[9] color measures the relative strengths of the star and the dominant dust component, amorphous carbon, which has an opacity that is a smooth function of wavelength with no known resonances (e.g., Jura 1983, 1986; Martin \& Rogers 1987). The color serves as a proxy for the dust production rate (Groenewegen et al. 2007) and is determined using two regions of

\footnotetext{
$\overline{11}$ https://www.sofia.usra.edu/sites/default/files/Uspot_DCS_DPS / Documents/Known_Issues_010518.pdf
} 


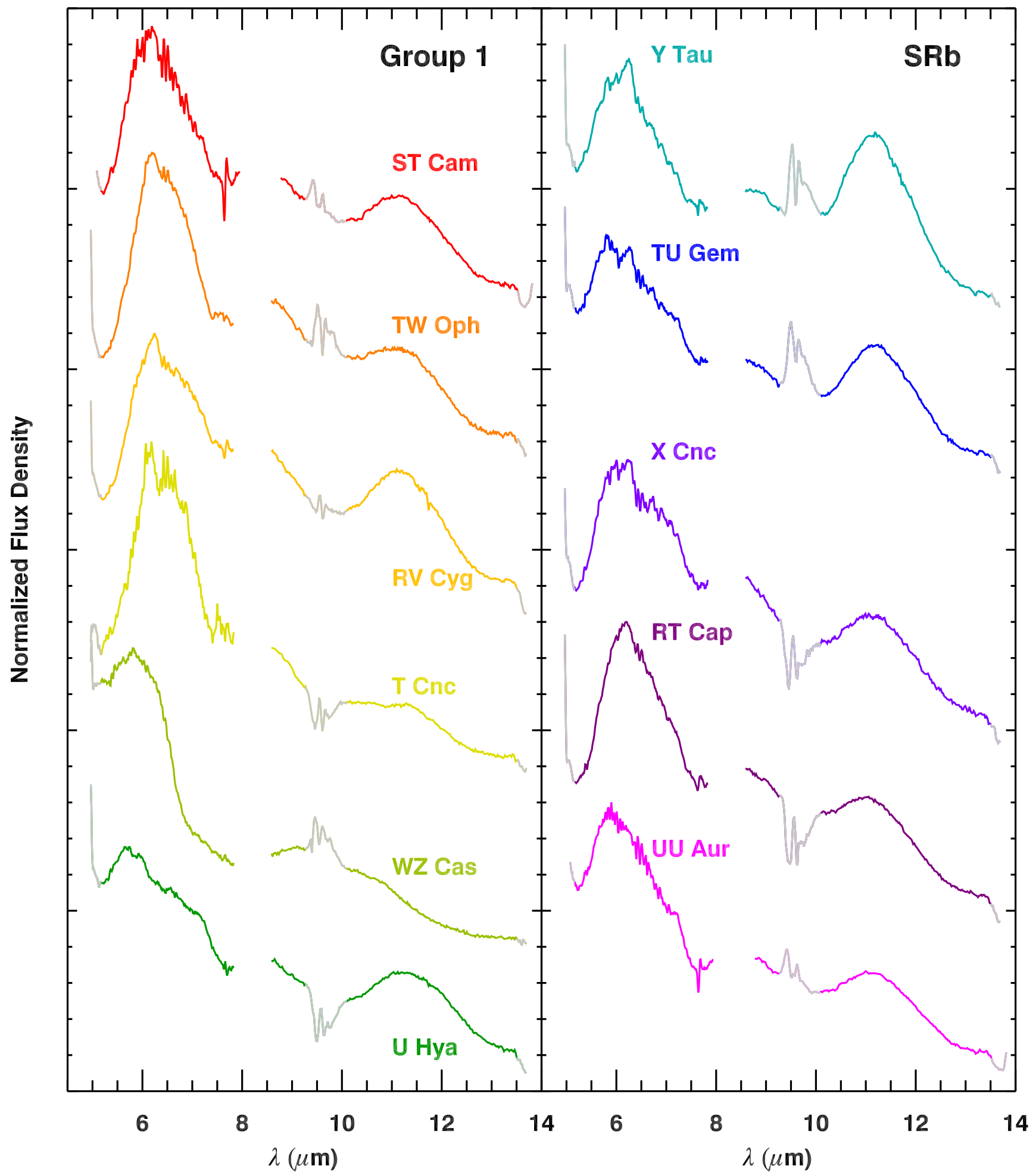

Figure 2. Group 1: SRb variables, $F_{\nu}(12 \mu \mathrm{m})>40 \mathrm{Jy}$. Segments in gray are contaminated by telluric ozone $(9.25-10.1 \mu \mathrm{m})$ or instrumental artifacts $(<5.17$ and $>13.5 \mu \mathrm{m}$ ). A telluric residual is also sometimes present at 7.6-7.7 $\mu \mathrm{m}$. Spectra have been normalized and offset for clarity.

the spectra that are free of molecular bands and solid-state features.

Due to residuals from the telluric ozone feature in the SOFIA spectra, the wavelengths used to define these features had to be adjusted compared to those used by Sloan et al. (2016). In order to compare apples to apples, we re-extracted the spectral features from the Magellanic (IRS) and Galactic (SWS) samples using the revised wavelength ranges. The Appendix gives the new values, along with the updated wavelength ranges. Although the exact values for a given star have shifted slightly compared to Sloan et al. (2016), the overall characteristics of the samples remain the same. Thus, we will refer to the color as [6]-[9] for simplicity when comparing to values in the literature.

Table 2 gives the results for the [6]-[9] color and the relative $\mathrm{SiC}$ strength for the SOFIA sample. The parameter $\lambda_{C}$ is the 
wavelength at which half of the flux in the $\mathrm{SiC}$ feature is to the red and half to the blue. The table includes the infrared spectral classification by Sloan et al. (2016), which is based on the [6][9] color and the dust production rate (DPR or $\dot{D}$ ). $\dot{D}$ is calculated from the [6]-[9] color, assuming an outflow velocity $v_{\text {out }}$ of $10 \mathrm{~km} \mathrm{~s}^{-1}$ according to the formula ${ }^{12}$

$$
\begin{aligned}
\log \dot{D}\left(\frac{M_{\odot}}{\mathrm{yr}}\right)= & \log \left(\frac{v_{\text {out }}}{10 \mathrm{~km} \mathrm{~s}^{-1}}\right) \\
& -8.9+1.6([6]-[9]) .
\end{aligned}
$$

We also can identify the presence of either $\mathrm{C}_{2} \mathrm{H}_{2}$ or CS absorption bands in many of the spectra between $\sim 7$ and $7.6 \mu \mathrm{m}$ (rarely both at the same time). The $v_{4}+v_{5} \mathrm{C}_{2} \mathrm{H}_{2}$ bands can be recognized by eye from the central peak of classic "W" shape at $\sim 7.5 \mu \mathrm{m}$, while a "knee" in the spectra reveals the CS $\Delta v=1$ bandhead at $\sim 7.3 \mu \mathrm{m}$. To characterize these absorption bands, we use a procedure similar to the Manchester method, with the blue end of the extraction set at $6.9 \mu \mathrm{m}$ and the red end at $7.57 \mu \mathrm{m}$. These wavelengths cover only half of the $\mathrm{C}_{2} \mathrm{H}_{2}$ band, but they avoid the telluric residual at 7.6-7.7 $\mu \mathrm{m}$ and gap in coverage beyond $7.8 \mu \mathrm{m}$. Thus, we determine a partial equivalent width, $\mathrm{EW}_{p}$, and the wavelength centroid for the partial band, $\lambda_{p}$. We stress that $\mathrm{EW}_{p}$ and $\lambda_{p}$ are used here only as diagnostics because they only cover part of the bands. We apply the same wavelength stops defined for the FORCAST data to the SWS data as well.

Figure 6 (left) shows examples of FORCAST spectra that have absorption either from CS or from $\mathrm{C}_{2} \mathrm{H}_{2}$, as well as a spectrum without a strong feature from either molecule (although absorption is clearly present in the general wavelength region). The right-hand panel shows similar spectra from the SWS sample, with the CS, and especially the $\mathrm{C}_{2} \mathrm{H}_{2}$ feature, more fully sampled. Many sources also show absorption below $6 \mu \mathrm{m}$, likely from $\mathrm{CO}$ and/or $\mathrm{C}_{3}$ (e.g., Jørgensen et al. 2000), but the limited wavelength coverage prevents further analysis with the FORCAST spectra here.

\subsection{SiC Contrast and [6]-[9] Color}

Figure 7 shows the ratio of the $\mathrm{SiC}$ dust feature to the continuum as a function of [6]-[9] color, which traces the amorphous carbon content, with the SOFIA sample in the top panel. A boundary at [6]-[9] $\sim 0.3$ cleanly separates the SRb variables (Group 1) and Miras (Groups 2 and 3). In the SRbs to the blue of that boundary, the relative strength of the SiC dust feature rises steeply with increasing color, while in the Miras to the red, it decreases more gradually as the color grows redder, with the longer-period Miras associated with redder [6]-[9] colors.

The lower panel in Figure 7 adds the SWS sample of Galactic carbon stars, colored by group as with the SOFIA sample. The boundaries and differences in behavior of the SRbs and Miras remain unchanged.

Figure 8 compares the Galactic sample to those in the LMC and the SMC. In the lower two panels, sources not identified as semiregular or Mira variables in the OGLE-III survey (Soszyński et al. 2009, 2011) appear as brown symbols, and

\footnotetext{
12 Incorrect equations were given for the dust mass-loss rate by Sloan et al. (2008) and Sloan et al. (2012). The formula assumes optical constants from Rouleau \& Martin (1991); using those from Zubko et al. (2004) would give different values.
}

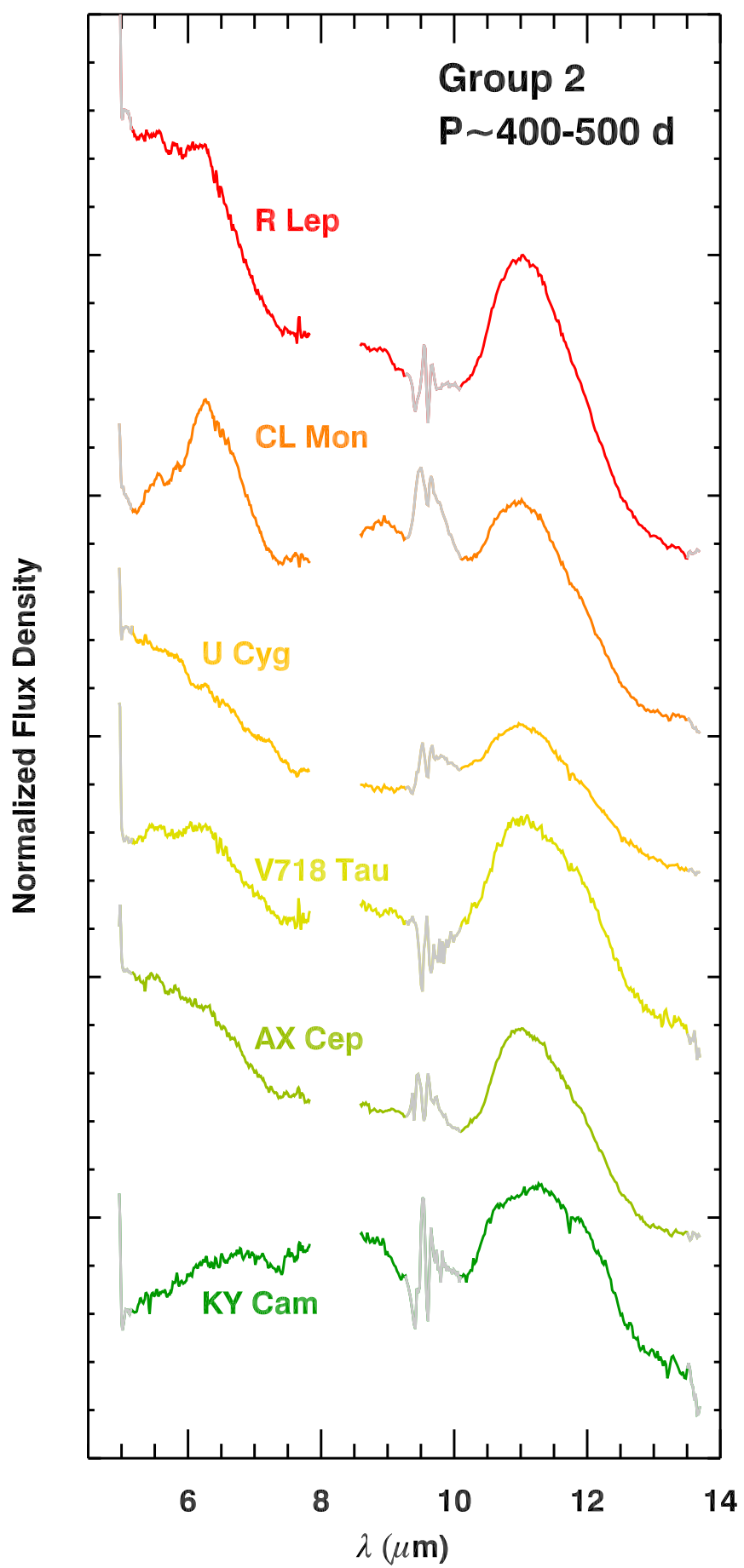

Figure 3. Group 2: Mira variables with 400 days $<P<500$ days and $F_{\nu}>60 \mathrm{Jy}$.

the Galactic carbon stars are plotted in gray for comparison. (LMC sources with [6]-[9] $>1.8$ are omitted here for clarity but are included in the table in the Appendix.)

For the LMC, all but 2 of the 13 semiregulars in the OGLE catalogs are on the same steeply rising segment as the Galactic $\mathrm{SRb}$ variables. The LMC Miras are split between the segment with the Galactic Miras and a lower segment with weaker $\mathrm{SiC} /$ continuum ratios for the same [6]-[9] color. The two segments rejoin at [6]-[9] $\sim 1.1$. The SMC sources generally follow the lower segment with weaker $\mathrm{SiC} /$ continuum ratios. 


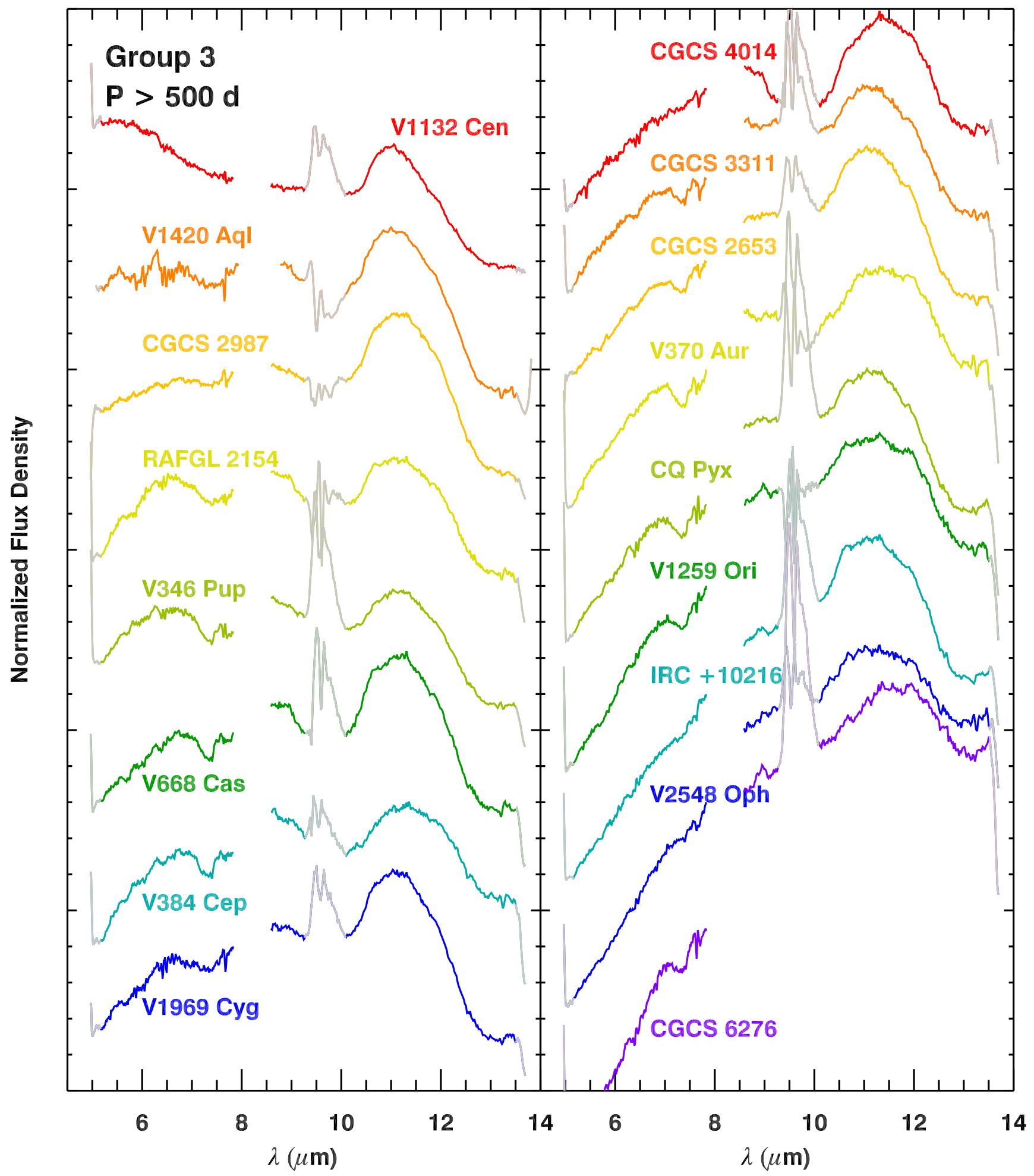

Figure 4. Group 3: Miras with $P>550$ days and $F_{\nu}>150 \mathrm{Jy}$.

\subsection{Molecular Gas Absorption}

The G063 grism of FORCAST covers the 4.9-7.8 $\mu \mathrm{m}$ range, which for carbon stars can include absorption bands from carbonbearing molecules such as $\mathrm{C}_{2} \mathrm{H}_{2}, \mathrm{HCN}, \mathrm{CS}, \mathrm{C}_{3}$, and $\mathrm{CO}$ (e.g., Goebel et al. 1980, 1981; Aoki et al. 1998, 1999; Jørgensen et al. 2000; Speck et al. 2006, and references therein). The Galactic spectra typically show either $\mathrm{C}_{2} \mathrm{H}_{2}$ or $\mathrm{CS}$ absorption most prominently. Figure 9 plots the partial equivalent width of the absorption band $\left(\mathrm{EW}_{p}\right)$ against the apparent centroid of that part of the band visible in the FORCAST spectra $\left(\lambda_{p}\right)$. The figure also includes the SWS sample, with the spectra analyzed with the same wavelength stops as the FORCAST spectra. The majority of the CS sources are in the lower left quadrant, with $\lambda_{p}<7.25 \mu \mathrm{m}$ and $\mathrm{EW}_{p}<0.005$. The $\mathrm{C}_{2} \mathrm{H}_{2}$ sources, in contrast, have $\lambda_{p}>7.2 \mu \mathrm{m}$ and $\mathrm{EW}_{p}>0$, and generally lie in the upper right quadrant. As noted before by Zijlstra et al. (2006) and Matsuura et al. (2006), 


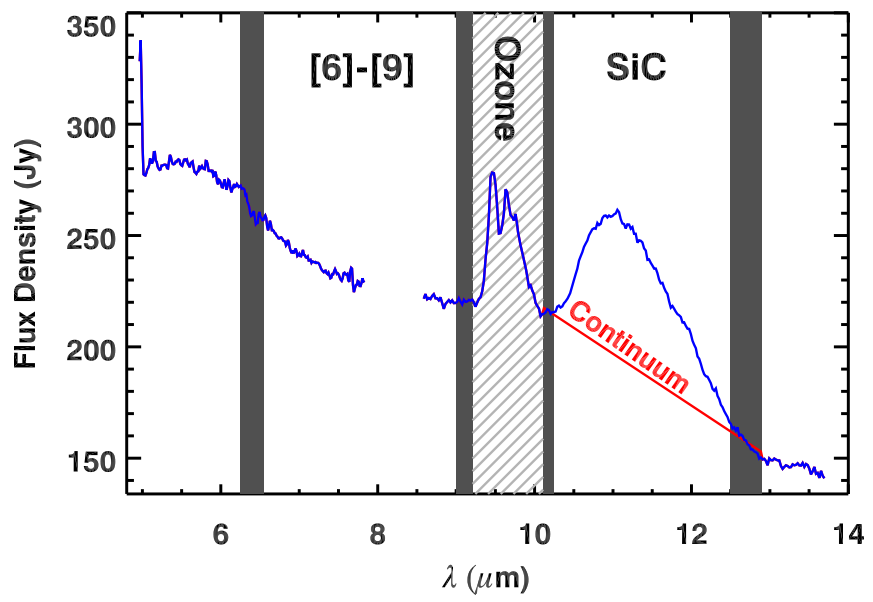

Figure 5. Feature extraction example with V1132 Cen. The [6]-[9] color is measured from the leftmost gray bands. The silicon carbide feature using the rightmost gray bands and the red line for the continuum level. The gray-striped region is contaminated by telluric ozone.

the Magellanic samples do not contain any sources with clear CS features. The vast majority would be in the upper right.

The right-hand panel of Figure 9 shows the same data but color coded by the variability type. Almost all of the Miras are in the upper right region due to strong $\mathrm{C}_{2} \mathrm{H}_{2}$ bands. The SRbs are split between the $\mathrm{CS}$ and $\mathrm{C}_{2} \mathrm{H}_{2}$ regions. Table 3 summarizes the distribution of the bands between the Miras and SRbs for the Galactic sources.

\section{Discussion}

\subsection{Molecular Gas}

The Galactic carbon stars observed with the SWS and the FORCAST grisms show a distinct difference between Miras and semiregular variables in their molecular gas features. While $\mathrm{C}_{2} \mathrm{H}_{2}$ dominates the molecular absorption in the Miras, CS dominates in about half of the SRb variables.

The Magellanic sample of carbon stars observed with the IRS do not show this dependence of molecular chemistry on variability type. Instead, $\mathrm{C}_{2} \mathrm{H}_{2}$ dominates the molecular absorption, with no CS bands apparent, no matter the variability type, as first noticed in the LMC by Zijlstra et al. (2006) and Matsuura et al. (2006).

Matsuura et al. (2006) suggested that the $\mathrm{C}_{2} \mathrm{H}_{2}$ band could mask the CS band in the Magellanic sample, but the bands occur at different wavelengths and the CS should be detectable if it were present. In addition, we would expect a continuous distribution of relative strengths in the two bands in the Galactic sample, but Figure 9 shows a bimodal distribution.

The lack of visible CS bands in the Magellanic spectra likely arises from differences in metallicity and lower abundances of sulfur. Metallicity differences can also explain the rising strength of the $\mathrm{C}_{2} \mathrm{H}_{2}$ bands from the Milky Way to the LMC to the SMC (e.g., van Loon et al. 1999; Matsuura et al. 2002; Sloan et al. 2006). The progressively lower oxygen abundances in the LMC and $\mathrm{SMC}$ lead to higher $\mathrm{C} / \mathrm{O}$ ratios after dredge ups. This in turn produces stronger $\mathrm{C}_{2} \mathrm{H}_{2}$ bands, as noted previously for bands in the 5-8 $\mu \mathrm{m}$ region and $\sim 14 \mu \mathrm{m}$ (e.g., Sloan et al. 2006; Zijlstra et al. 2006), and in the 3-4 $\mu$ m region (e.g., van Loon et al. 1999; Matsuura et al. 2002, 2005; van Loon et al. 2006).

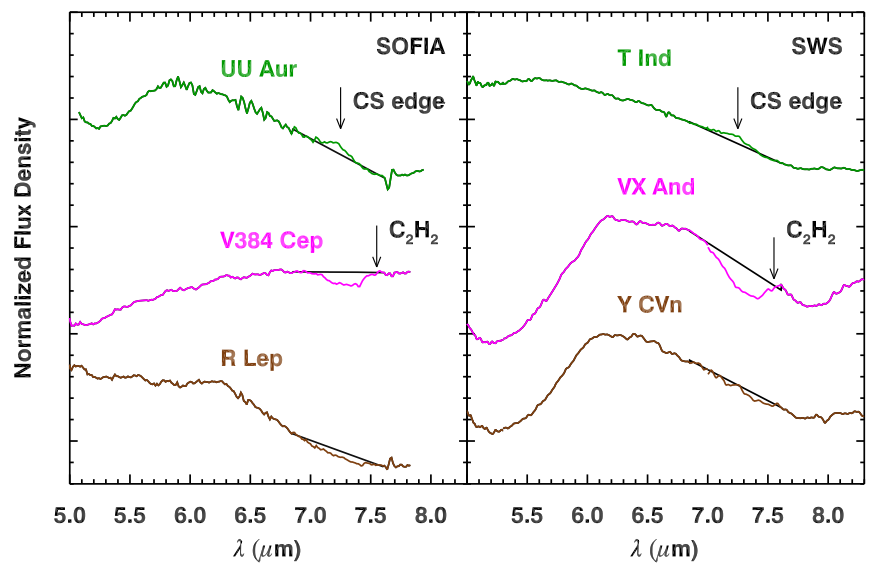

Figure 6. The 6.5-8 $\mu \mathrm{m}$ absorption complex. (Left) Examples from the SOFIA data with (top) the $\mathrm{CS}$ absorption band, (middle) the $\mathrm{C}_{2} \mathrm{H}_{2}$ band, and (bottom) neither band apparent; (right) SWS examples. Arrows indicate the features assessed by eye; the black lines show the feature extraction region.

The addition of the FORCAST spectra to the Galactic carbon star sample reveals that with a single exception, ${ }^{13} \mathrm{CS}$ only appears in the Galactic SRbs, and then only in some of them (Table 3). Thus, the presence of CS requires that the carbon star be (1) metal-rich and (2) pulsating as an SRb variable. However, only half of the semiregular variables in the Galactic sample show CS bands, so these conditions are necessary, but not sufficient.

\subsection{Dust and Metallicity}

The differences in the behavior of the SiC dust emission as a function of [6]-[9] color between the Milky Way and Magellanic Clouds are readily apparent in Figure 8. Previous works, starting with Sloan et al. (2006) and Zijlstra et al. (2006), largely investigated these differences through the lens of metallicity. The metallicities in the Magellanic Clouds are typically in the range $[\mathrm{Fe} / \mathrm{H}] \sim-0.7$ to -0.3 for the LMC (e.g., Piatti \& Geisler 2013) and $[\mathrm{Fe} / \mathrm{H}] \sim-1.5$ to -0.5 in the SMC (e.g., Piatti 2012; Rubele et al. 2015), and fall even lower for dwarf spheroidals in the Local Group (e.g., Sloan et al. 2012, and references therein). Differences in $\mathrm{Si}$ abundances could explain why the relative contribution of $\mathrm{SiC}$ dust builds only slowly as the dust increases in the SMC and other metal-poor galaxies in the Local Group, while in the Galaxy, it rises much more rapidly before the continued formation of amorphous carbon overwhelms it.

\subsection{Dust and Variability Type}

With the expanded Galactic sample, we can now revisit the dust properties in terms of the variability types. As already noted, a sharp color boundary separates the Galactic semiregulars and Miras in Figure 7. The Magellanic samples observed with the IRS show a similar dichotomy, with only a handful of exceptions (Figure 8).

Figure 10 shows related behavior for a much larger photometric sample of carbon stars in the LMC from the OGLE-III survey (Soszyński et al. 2009). The top panel presents a period-luminosity diagram $(P-L)^{14}$ for the stars identified as carbon-rich Miras or semiregular variables, with the semiregulars color coded according to whether they appear

\footnotetext{
13 U Cyg, a Mira in Group 2, is the lone exception.

14 Pioneered by Leavitt \& Pickering (1912).
} 
Table 2

Dust Properties

\begin{tabular}{|c|c|c|c|c|c|}
\hline $\begin{array}{l}\text { Star } \\
\text { Name }\end{array}$ & $\begin{array}{c}{[6]-[9]} \\
(\mathrm{mag})\end{array}$ & $\begin{array}{c}F_{\mathrm{SiC}} / \\
\text { Continuum }\end{array}$ & $\begin{array}{c}\lambda_{C} \\
(\mu \mathrm{m})\end{array}$ & $\left(\begin{array}{c}\log \dot{D} \\
\left(M_{\odot} \mathrm{yr}^{-1}\right)\end{array}\right.$ & $\begin{array}{c}\text { CE } \\
\text { Class }\end{array}$ \\
\hline \multicolumn{6}{|c|}{ Group 1} \\
\hline ST Cam & $0.180 \pm 0.010$ & $0.187 \pm 0.009$ & $11.30 \pm 0.07$ & -8.61 & 1 \\
\hline Y Tau & $0.287 \pm 0.014$ & $0.368 \pm 0.004$ & $11.25 \pm 0.01$ & -8.44 & 1 \\
\hline TU Gem & $0.281 \pm 0.010$ & $0.266 \pm 0.004$ & $11.29 \pm 0.02$ & -8.45 & 1 \\
\hline U Hya & $0.285 \pm 0.006$ & $0.206 \pm 0.005$ & $11.37 \pm 0.04$ & -8.44 & 1 \\
\hline TW Oph & $0.100 \pm 0.008$ & $0.173 \pm 0.003$ & $11.30 \pm 0.02$ & -8.74 & 1 \\
\hline RT Cap & $0.178 \pm 0.010$ & $0.174 \pm 0.002$ & $11.24 \pm 0.01$ & -8.62 & 1 \\
\hline RV Cyg & $0.252 \pm 0.010$ & $0.233 \pm 0.003$ & $11.28 \pm 0.02$ & -8.50 & 1 \\
\hline \multicolumn{6}{|c|}{ Group 2} \\
\hline U Cyg & $0.433 \pm 0.005$ & $0.169 \pm 0.002$ & $11.22 \pm 0.02$ & -8.21 & 2 \\
\hline AX Cep & $0.448 \pm 0.005$ & $0.347 \pm 0.006$ & $11.22 \pm 0.02$ & -8.18 & 2 \\
\hline \multicolumn{6}{|c|}{ Group 3} \\
\hline V668 Cas & $0.835 \pm 0.006$ & $0.207 \pm 0.003$ & $11.28 \pm 0.02$ & -7.56 & 3 \\
\hline V370 Aur & $1.054 \pm 0.003$ & $0.115 \pm 0.004$ & $11.42 \pm 0.06$ & -7.21 & 4 \\
\hline V1259 Ori & $1.263 \pm 0.006$ & $0.104 \pm 0.003$ & $11.37 \pm 0.05$ & -6.88 & 5 \\
\hline CGCS 6276 & $1.526 \pm 0.008$ & $0.062 \pm 0.003$ & $11.50 \pm 0.08$ & -6.46 & 3 \\
\hline V346 Pup & $0.765 \pm 0.004$ & $0.161 \pm 0.003$ & $11.34 \pm 0.03$ & -7.68 & 3 \\
\hline CQ Pyx & $1.139 \pm 0.004$ & $0.130 \pm 0.003$ & $11.33 \pm 0.03$ & -7.08 & 4 \\
\hline IRC +10216 & $1.265 \pm 0.006$ & $0.146 \pm 0.003$ & $11.31 \pm 0.03$ & -6.88 & 5 \\
\hline CGCS 2653 & $1.044 \pm 0.002$ & $0.157 \pm 0.003$ & $11.30 \pm 0.03$ & -7.23 & 4 \\
\hline
\end{tabular}

Note. Uncertainties are $1 \sigma$ and primarily reflect the observational noise in the spectra.

on the first overtone or the fundamental-mode sequence (e.g., Wood \& Sebo 1996; Wood et al. 1999; Fraser et al. 2005). The bottom panel uses the same color codes and maps the stars into color-color space using photometry from the Spitzer SAGE project (Meixner et al. 2006) and the WISE mission (Wright et al. 2010). ${ }^{15}$ The carbon stars in the SMC behave nearly identically (Sloan et al. 2015).

The color-color diagram reveals a clear dichotomy between the semiregulars and Miras. The carbon stars identified in the OGLE-III survey as semiregulars can pulsate in either an overtone or fundamental mode (Soszyński et al. 2009, 2013), and thus appear in two separate sequences in the $P-L$ diagram. However, nearly all of them follow the same sequence in color-color space. The Mira variables follow a different sequence. Since amorphous carbon dominates the dust around carbon stars, adding more dust to a carbon star will redden it

\footnotetext{
15 SAGE: Surveying Agents of Galaxy Evolution; WISE: Wide-field Infrared Survey Explorer.
}

Table 3

CS, $\mathrm{C}_{2} \mathrm{H}_{2}$ Absorption-Galactic Sample

\begin{tabular}{lccc}
\hline \hline & \multicolumn{3}{c}{ No. of Stars Showing } \\
\cline { 2 - 4 } & $\mathrm{C}_{2} \mathrm{H}_{2}$ & $\mathrm{CS}$ & Other \\
\hline $\mathrm{SRb}$ & $6(26 \%)$ & $12(52 \%)$ & $5(22 \%)$ \\
Mira & $27(64 \%)$ & $1(2 \%)$ & $14(33 \%)$ \\
\hline
\end{tabular}

monotonically in all broadband near-infrared and mid-infrared colors, leading to a readily recognizable sequence in any colorcolor space (e.g., Sloan 2017, Sloan et al. 2019).

The [3.6]-[4.5] colors of the semiregulars generally stay below 0 , although they show a range of [5.8]-[8] colors. The relatively blue colors of the semiregulars in all other infrared colors indicate that there is too little dust to explain their behavior in [5.8]-[8]. Sloan et al. (2015) argued that the $\mathrm{C}_{3}$ absorption band at $\sim 5 \mu \mathrm{m}$ is responsible for the observed behavior; other molecules, such as $\mathrm{CO}$, should also affect the $5.8 \mu \mathrm{m}$ filter. In the dustier stars, the 


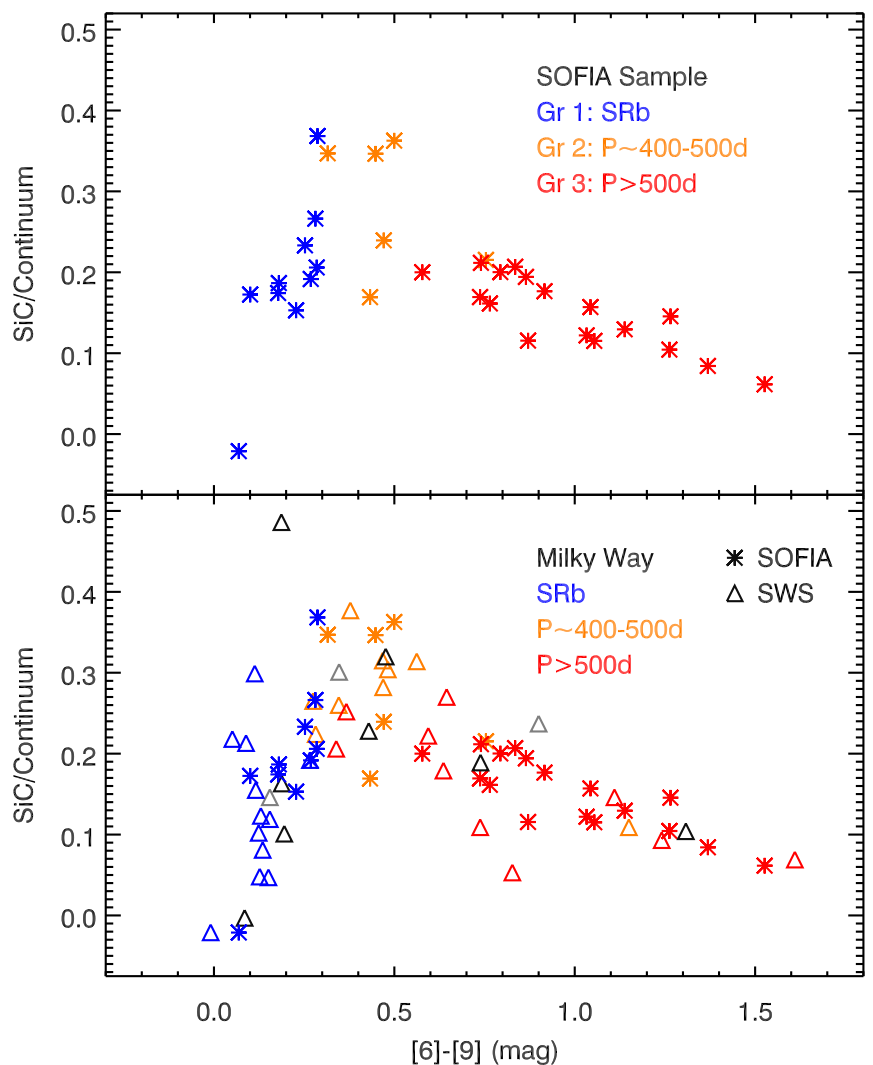

Figure 7. [6]-[9] vs. SiC strength for the Milky Way. (Top) The SOFIA sample, with colors corresponding to the selection group. (Bottom) The SWS sample added, also colored by group (gray triangles are SRas, black triangles are Lbs or unknown).

effect of any molecular absorption bands in this spectral region will diminish due to dust veiling.

The additional Galactic carbon stars observed with SOFIA have revealed a second dependence of dust characteristics on the variability type. The rapid rise of the relative strength of the $\mathrm{SiC}$ emission feature with increasing overall dust content is limited to the semiregulars. Once a star begins pulsating in the fundamental mode as a Mira, the amorphous dust content climbs, pushing the [6]-[9] color beyond the boundary at $\sim 0.3$. The relative strength of the $\mathrm{SiC}$ dust, however, turns over and diminishes.

\subsection{Variability Type and Pulsation Mode}

Comparison of the Galactic and Magellanic samples is hampered by differences in how their variability is classified. Pulsating giants in the Galaxy have been classified for several decades as Miras if their pulsation amplitude (peak to peak) exceeds 2.5 mag at $V$ (e.g., Mattei et al. 1997; Samus et al. 2017). ${ }^{16}$ The semiregular variables on the AGB have lower amplitudes. Those with fairly stable lightcurves are classified as SRa variables, while the SRbs are distinguished by more complex lightcurves that still show a discernible periodicity some of the time (e.g., Glasby 1968; Hoffmeister et al. 1984).

The OGLE-III survey separated Miras from semiregulars in the LMC at an amplitude of $\Delta I=0.8$ mag (Soszyński et al. 2009). Miras pulsate in the fundamental mode (Wood \& Sebo 1996). The OGLE-III survey did not subdivide the semiregulars, though, unlike many studies of Galactic variables. Figure 10 shows that

\footnotetext{
${ }^{16}$ Hoffmeister et al. (1984) noted that some observers use a limit of 2 mag, and Payne-Gaposchkin \& Gaposchkin (1938) quoted $1.5 \mathrm{mag}$.
}

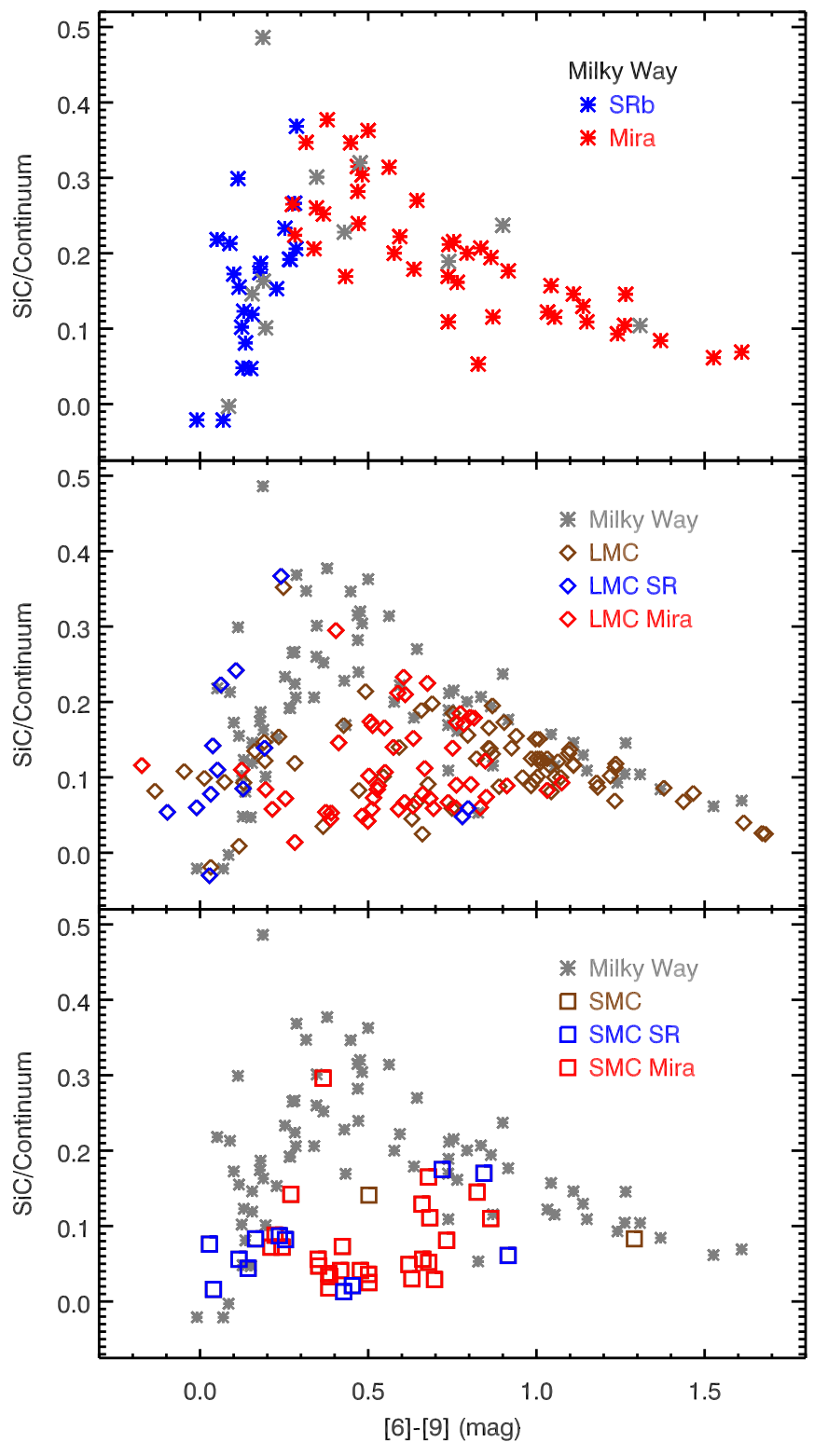

Figure 8. [6]-[9] vs. SiC strength. (Top) The combined Galactic sample, with SRbs in blue and Miras in red; gray asterisks are Galactic SRas, Lbs, or unknown. (Middle) The LMC sample; sources with no OGLE class are in brown; Galactic sources are gray asterisks. (Bottom) The SMC sample, same colors as for the LMC.

the dominant pulsation mode in semiregulars can be either the fundamental mode or first overtone, as has been noted previously with shorter-wavelength data (e.g., Wood et al. 1999).

Thus, we have two groups of carbon-rich semiregular variables in the LMC, and two in the Galaxy. The Galactic SRas have lightcurves with well-defined periodicities but weak amplitudes, making them the Galactic analogs of the fundamental-mode semiregulars in the LMC. Similarly, Galactic SRbs are overtone pulsators. Using Gaia distances for carbon stars has proven challenging so far (e.g., McDonald et al. 2018), so placing the $\mathrm{SRa}$ and $\mathrm{SRb}$ variables on a $P-L$ diagram is problematic.

We can still compare the periods, though. Figure 11 shows that the distribution of periods for the SRb, SRa, and Mira variables in the Galaxy behave very much like the overtone semiregulars, fundamental-mode semiregulars, and Miras in the LMC, respectively. For the Galactic sample, we cross-referenced 


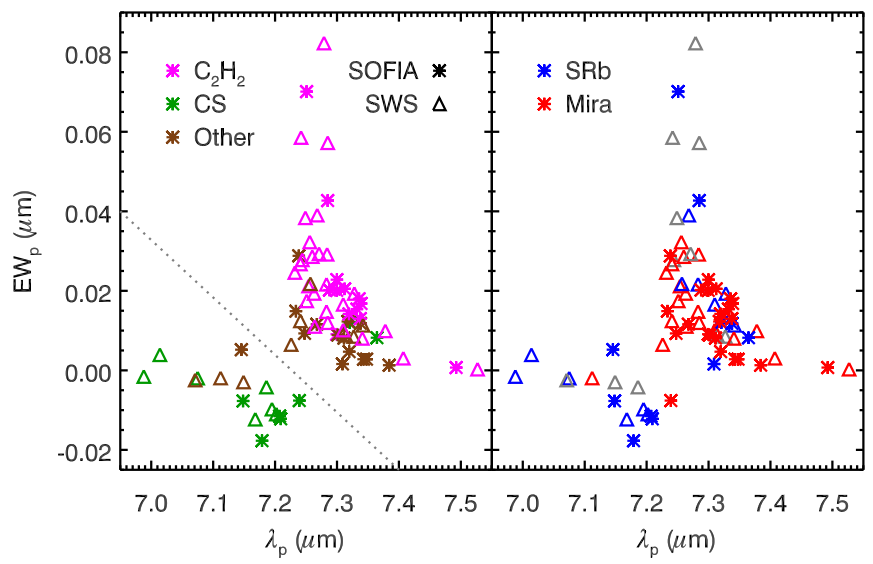

Figure 9. Partial equivalent width vs. feature centroid. (Left) Sources color coded by the qualitative assessment. Almost all the CS sources (green) are in the lower left quadrant and the $\mathrm{C}_{2} \mathrm{H}_{2}$ sources (magenta) are in the upper right quadrant. (Right) Sources color coded by variability. The Miras are almost entirely in the $\mathrm{C}_{2} \mathrm{H}_{2}$ region, while the SRbs are split between $\mathrm{CS}$ and $\mathrm{C}_{2} \mathrm{H}_{2}$. The dashed line in the left panel is simply to guide the eye; gray symbols are the SRas, Lbs, and unknowns, as in Figure 8.

the Catalog of Galactic Carbon Stars (3rd ed.; Alksnis et al. 2001) with the General Catalog of Variable Stars (GCVS 5.1; Samus et al. 2017), matching sources within $10^{\prime \prime}$. The quantitative differences in the distributions likely arise from the differences in the initial mass limits for carbon stars in the LMC and Galaxy, which in turn limit the range of luminosities a star will show on the AGB. ${ }^{17}$ The $P-L$ relation ties these luminosities to the pulsation periods of the stars.

The qualitative conclusion remains clear: the first overtone dominates the pulsation of the SRb variables, while the fundamental mode has grown stronger in the SRa variables. Once the fundamental mode dominates all other modes and the amplitude grows strong enough, the star becomes a Mira variable.

\subsection{Pulsation and Dust}

We can now relate the differences in dust quantity and composition illustrated in Figures 7 and 8 with the pulsation mode of the central star. Stars with weak pulsations do not produce significant quantities of dust. Whether their pulsations are dominated by an overtone mode or the fundamental, if their amplitudes are less than $\sim 2.5 \mathrm{mag}$ in $V$, their [6]-[9] colors remain below 0.3, always in the Galaxy and most of the time in the Magellanic Clouds. For most of the carbon-rich semiregulars in the LMC and all in the Galaxy, a slight increase in total dust content is accompanied by a sharp increase in the amount of $\mathrm{SiC}$ dust. Once the fundamental pulsation mode dominates the star and its pulsation amplitude grows strong enough for it to be classified as a Mira, the total dust content takes off, and the relative strength of the $\mathrm{SiC}$ feature diminishes.

To rephrase, the key to significant dust production on the AGB is not just the pulsation mode, but also the pulsation amplitude of the central star (e.g., Mattsson et al. 2008; McDonald \& Trabucchi 2019). These strong pulsations are sufficient to push molecular gas far enough from the stellar photosphere so that amorphous carbon can condense in the quantities necessary for radiation pressure on the dust to drive significant mass loss (e.g., McDonald et al. 2018; McDonald \& Trabucchi 2019). The forming dust will incorporate much of

\footnotetext{
17 That is, the lowest mass carbon stars in the LMC remain oxygen-rich in the Galaxy.
}
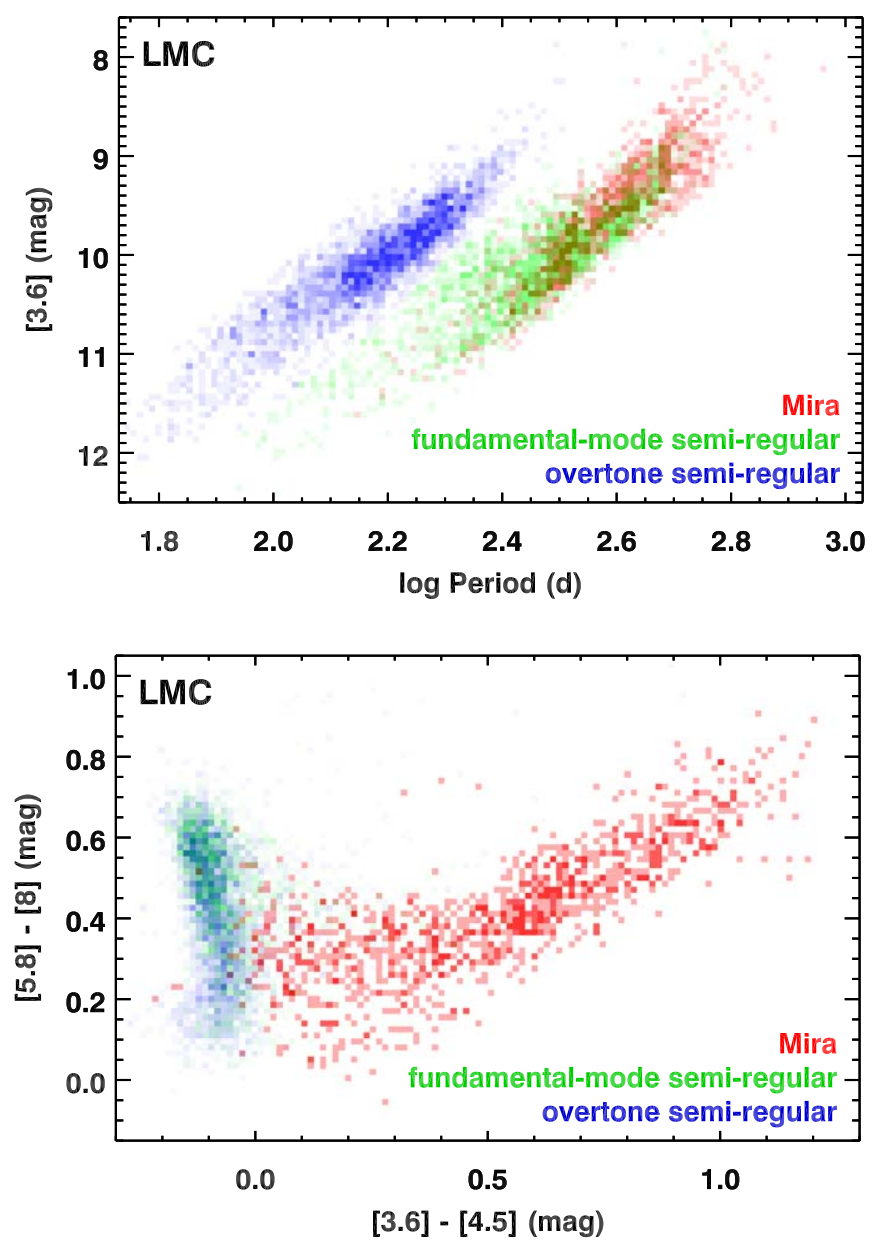

Figure 10. LMC carbon stars. (Top) Period-luminosity diagram for carbon stars identified as semiregular variables on the first overtone sequence (blue), semiregulars on the fundamental sequence (green), and Miras (red). (Bottom) Mid-infrared color-color diagram using the same color codes as the $P-L$ diagram.

the carbon-rich molecular gas in the outflows and veil the absorption from those that are left, which will weaken the molecular absorption as the dust content grows.

When overtone modes dominate the fundamental mode, the pulsation amplitude remains weak, and the star is unable to drive significant dust production or mass loss. In these cases, the [6]-[9] color is limited by the lack of overall dust, and the $\mathrm{SiC}$ dust emission feature is relatively strong.

The semiregular variables on the fundamental-mode sequence may be of too low an initial mass to drive strong pulsations. Alternatively, they may have transitioned to this sequence too recently and have not yet had time for their pulsations to grow enough to trigger dust production. Thus, their dust properties often still look like the semiregulars on the first overtone (SRbs) even though their pulsation periods and luminosities place them on the fundamental sequence with the Miras in the $P-L$ diagrams.

\subsection{Grain Scenarios}

Lagadec et al. (2007) suggested that the two sequences of $\mathrm{SiC}$ versus total dust (Figure 8) differ in part due to the metallicity dependence of the condensation temperatures of $\mathrm{SiC}$ and graphite. Building on this idea, Leisenring et al. (2008) proposed that carbon-rich dust grains could form in layers (e.g., Kozasa et al. 1996; Lorenz-Martins et al. 2001), and the upper 


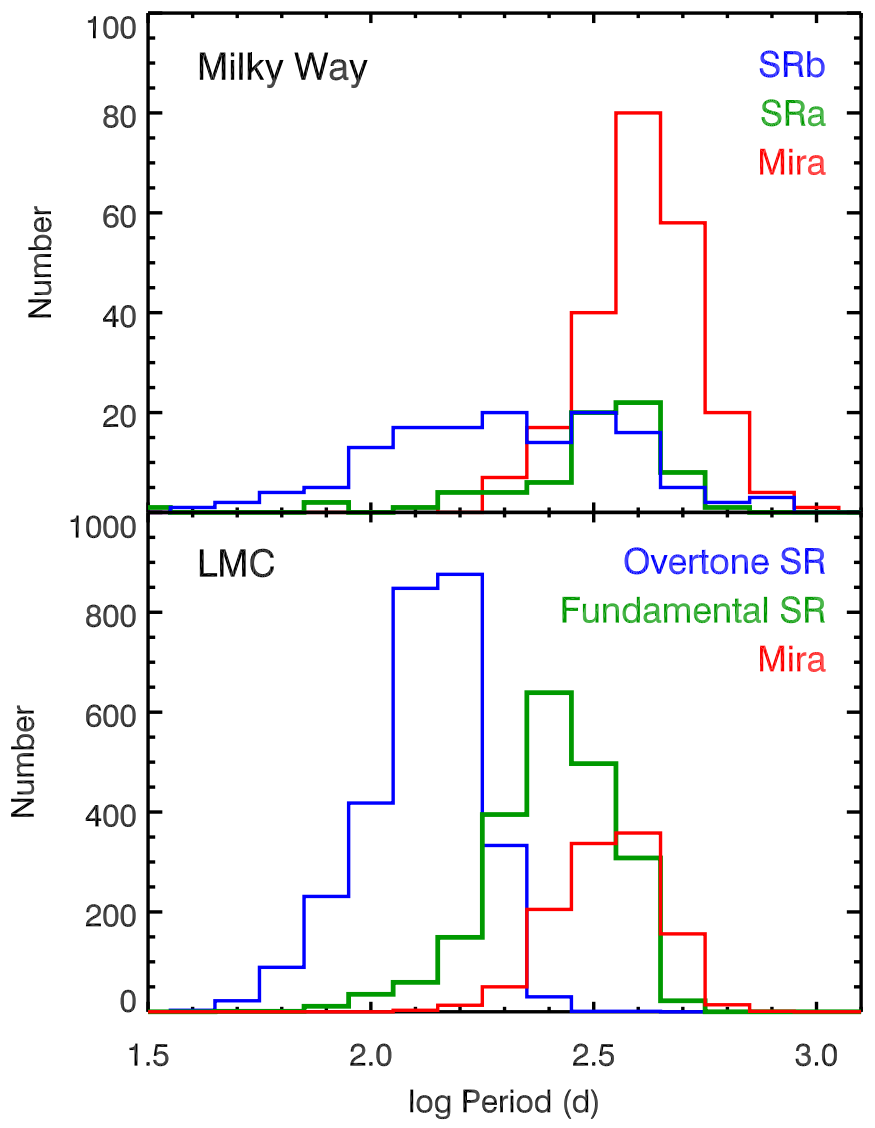

Figure 11. Period distributions. (Top) Galactic carbon stars classed as SRbs (blue), SRas (green), and Miras (red) in the GCVS. (Bottom) LMC carbon stars classed as SRVs on the first overtone sequence (green), SRVs on the fundamental-mode sequence (green), and Miras (red).

sequence resulted from the initial condensation of $\mathrm{SiC}$ dust which forms first (e.g., McCabe 1982; Ferrarotti \& Gail 2002; Dell'Agli et al. 2017; Nanni et al. 2019). These grains would then be covered by a layer of amorphous carbon, which progressively hides the $\mathrm{SiC}$ from the observer.

Another possibility should be considered. The $\mathrm{SiC}$ and amorphous carbon could form in separate populations of grains. In that case, the diminishing contribution of the SiC dust once stars start pulsating as Miras can be explained simply by the decreasing fraction of $\mathrm{SiC}$ dust as the condensation of amorphous carbon takes off. The relative abundances of carbon and silicon should make that result inevitable even without layering. As carbon is synthesized in the thermal pulses and silicon is not, the relative abundances become even more skewed as the star evolves and dredges up freshly fused carbon.

The infrared spectra provide no means of distinguishing whether the $\mathrm{SiC}$ and amorphous carbon grains are layered or form separately. In either scenario, the sharp boundary between the Miras and the semiregulars at [6]-[9] $~ 0.3$ and the dramatic difference in the behavior of the relative strength of the $\mathrm{SiC}$ feature on either side of that boundary point to a clear difference in the dust-formation mechanism between the Miras and semiregulars.

Croat et al. (2005) used isotopic abundances and the relative lack of s-process elements to show that $\mathrm{SiC}$ grains in meteorites condensed at an earlier stage in AGB evolution than other carbonaceous grains and under different conditions. Our finding that the $\mathrm{SiC}$ dust is produced predominantly by weakly pulsating carbon stars supports their conclusion. The different isotopic abundances in the $\mathrm{SiC}$ grains found by Croat et al. support the commonly assumed sequence of stars evolving from semiregulars to Miras.

\subsection{The Beginning of the End?}

The end of a star's life on the AGB must occur after it has lost a substantial fraction of its mass via a stellar wind driven by pulsation and radiation pressure on the condensing dust (e.g., Whitelock et al. 2003; McDonald et al. 2018; Bladh et al. 2019). The pulsations in the stellar interior can levitate material into the dust-formation zone, where the increased molecular and dust opacity allow the radiation to drive the wind (as first laid out by Jones et al. 1981, see also Höfner \& Olofsson 2018 and references therein). Which physical process triggers the pulsations that drive heavy mass loss, though, is still a matter of debate. For carbon stars, the $\mathrm{C} / \mathrm{O}$ ratio is an important parameter in the wind properties, although a better description may be that the total amount of excess free carbon is the key property (e.g., Lagadec \& Zijlstra 2008; Sloan et al. 2012; Eriksson et al. 2014; Nanni et al. 2017; Bladh et al. 2019). That is, the trigger would be a dredge-up event that pushes the $\mathrm{C} / \mathrm{O}$ ratio or the amount of free carbon over a critical threshold.

McDonald \& Trabucchi (2019), though, found that a transition between segments of the first overtone sequence, indicated by a larger $I$-band amplitude, was tied to significantly increased mass loss. Here, the dramatic change we observe in dust and gas properties between the first overtone semiregulars and the fundamental-mode Miras suggest that the transition of a carbon star to a Mira variable also triggers a substantial increase in dust production and total mass loss. This increase will rapidly strip its envelope and end its life on the AGB (e.g., Mattsson et al. 2008; McDonald et al. 2018).

The relations between the pulsational behavior of carbon stars and the properties of the gas and dust around them raise questions about oxygen-rich AGB stars, where the dust is usually dominated by silicates. For these stars, an infrared spectroscopic study comparing the overall dust quantity between the Miras and semiregulars analogous to what we have done in this paper has yet to be done. Differences in mineralogy have been found, though, and point to significantly different dust processing in the semiregulars (e.g., Sloan et al. 1996, 2003a; Uttenthaler et al. 2019).

Recent radio observations have revealed details on molecular line profiles and expansion velocities in the outer shell around both carbon-rich and oxygen-rich stars (e.g., McDonald et al. 2018; Díaz-Luis et al. 2019; Massalkhi et al. 2019, McDonald et al. 2019). Díaz-Luis et al. (2019), for example, have recently found that some oxygen-rich semiregulars have an unusual CO line profile inconsistent with spherical symmetry. This result needs to be confirmed in a larger sample, especially to confirm that it only appears in the oxygen-rich semiregulars. Additional observations and archival studies in both the infrared and radio are needed to better understand the relation between the pulsation of the central star, its chemistry, and how it ejects its envelope, forms dust, and evolves off of the AGB.

\section{Summary and Conclusions}

We observed 33 Galactic carbon stars from 5 to $13.8 \mu \mathrm{m}$ with SOFIA's FORCAST grisms. The sources were selected to expand the Galactic sample observed with the SWS on ISO and better align it with the Magellanic samples observed by the IRS 
on Spitzer. Using the Manchester system, we extracted the strengths of the dust features and equivalent widths for molecular gas absorption bands, with the wavelength ranges adjusted to avoid telluric features.

The new Galactic sample reveals multiple differences in the spectral properties between carbon-rich semiregular and Mira variables. The semiregulars in the sample include both SRb and SRa variables, in which the pulsations are dominated by the first overtone and fundamental mode, respectively. The Miras, also fundamental-mode pulsators, have much stronger pulsations than the SRas.

A [6]-[9] color of $\sim 0.3$ cleanly separates the Miras from the semiregulars. That color tracks the amount of amorphous carbon dust around the star and shows that the semiregulars have little circumstellar dust compared to the Miras.

The strength of the $\mathrm{SiC}$ dust feature at $\sim 11.3 \mu \mathrm{m}$ rises sharply in the semiregular variables. Once a star becomes a Mira, it begins to produce amorphous carbon dust in substantial quantities, and the $\mathrm{SiC}$ dust feature is masked as the overall dust content grows.

All of these differences between the semiregulars and Miras support the argument that strong fundamental-mode pulsations are required for significant rates of dust production in carbon stars. This statement holds for both the Galactic sample studied here and the Magellanic samples as well.

The 5-7.5 $\mu \mathrm{m}$ portion of the spectra can show absorption bands from $\mathrm{CS}$ and $\mathrm{C}_{2} \mathrm{H}_{2}$, but the CS is nearly always absent among the Miras, while it is present in half of the semiregulars. That is, CS only occurs in stars with weak pulsations, and then only in some of them. CS is not observed in Magellanic carbon stars regardless of their variability type; its presence must also require higher metallicity.

Lastly, for the Galactic sample, the clean separation of the Miras and semiregulars in [6]-[9] color points to a simple means of distinguishing these types of carbon-rich variables. Single-epoch infrared photometry or spectra can quantify the amount of dust around these stars and that makes it possible to identify the Miras without measuring their pulsation amplitudes or periods, both of which require observations of long temporal baselines, or placing them on a $P-L$ diagram, which requires knowing their distances.

We thank the anonymous referee whose careful reading and thoughtful suggestions have helped improve the paper. We also thank the SOFIA flight crew and staff scientists for making these observations. Based on observations made with the NASA/DLR Stratospheric Observatory for Infrared Astronomy (SOFIA). SOFIA is jointly operated by the Universities Space Research Association, Inc. (USRA), under NASA contract NNA17BF53C, and the Deutsches SOFIA Institut (DSI) under DLR contract 50 OK 0901 to the University of Stuttgart. Financial support for this work was provided by NASA through awards SOF03-0104 and SOF04-0129 issued by USRA. We made use of the NASA Astrophysics Data System, IRSA's Gator service, and CDS's Simbad and Vizier services.

Facilities: SOFIA (FORCAST), Spitzer (IRS), ISO (SWS).

\section{Appendix \\ Adjustments to Feature Extraction Wavelengths and Their Effects}

The Manchester method works as follows. The continuum is determined at feature-free wavelengths by averaging the flux in

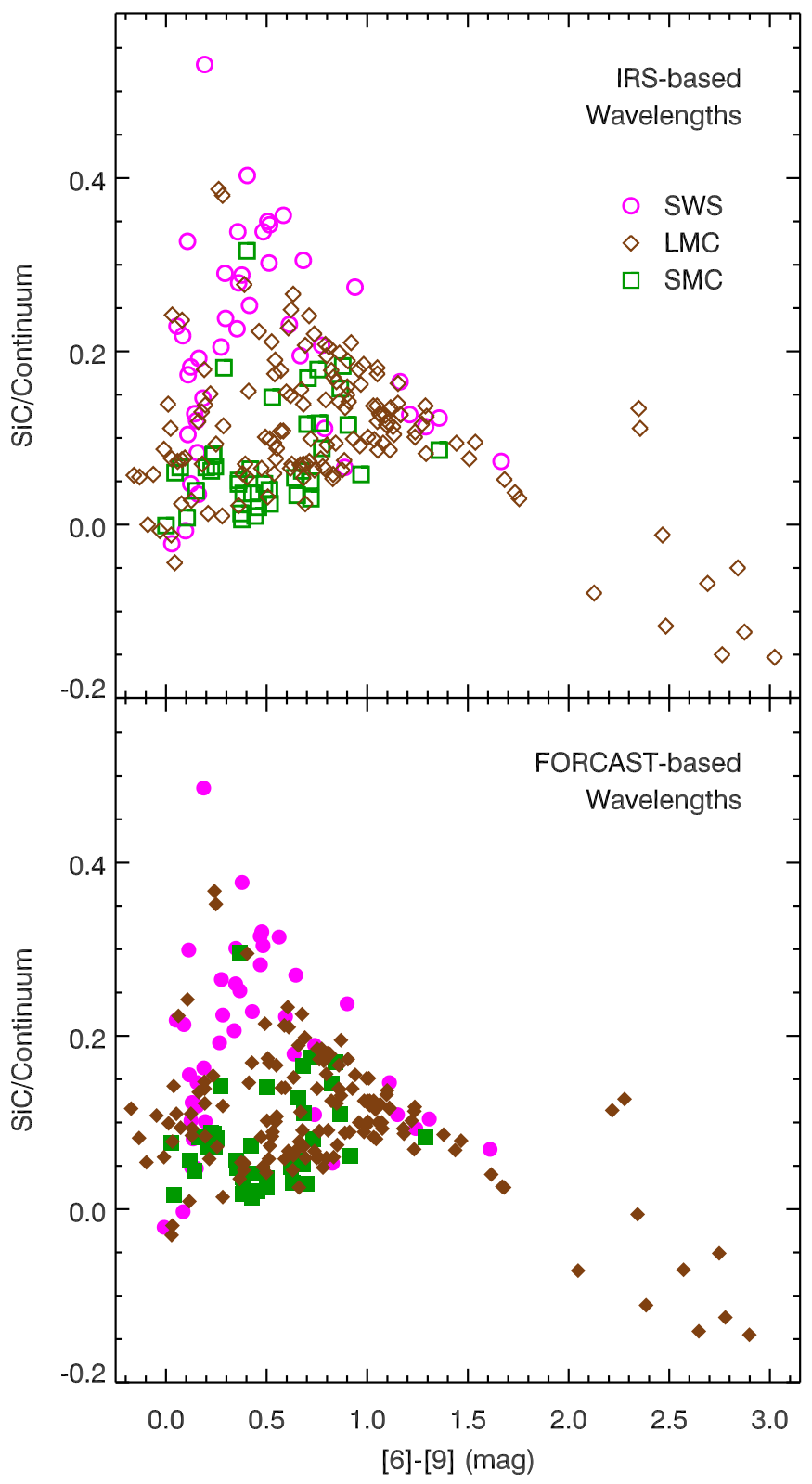

Figure 12. Comparisons of feature extractions using the FORCAST (FC) and IRS wavelength ranges. (Top) [6]-[9] vs. SiC/Cont. for the original, IRS-based ranges. (Bottom) [6]-[9] vs. SiC/Cont. for the new, FORCAST-based ranges. The magenta circles are the SWS sample; the brown diamonds are the IRS sample from the LMC; and the green squares are the IRS sample from the SMC.

Table 4

Extraction Range Comparison

\begin{tabular}{lcc}
\hline \hline & \multicolumn{2}{c}{$\lambda$ Range } \\
\cline { 2 - 3 } & \multicolumn{2}{c}{ Blue } \\
\hline Original & $6.25-6.55 \mu \mathrm{m}$ & \\
New & $6.25-6.55 \mu \mathrm{m}$ & $9.10-9.50 \mu \mathrm{m}$ \\
& \multicolumn{3}{c}{$\mathrm{SiC} /$ Continuum } & $9.00-9.21 \mu \mathrm{m}$ \\
\hline Original & $9.50-10.10 \mu \mathrm{m}$ & $12.50-12.90 \mu \mathrm{m}$ \\
New & $10.11-10.25 \mu \mathrm{m}$ & $12.50-12.90 \mu \mathrm{m}$ \\
\hline
\end{tabular}


Table 5

Revised Feature Strengths-SWS Sample

\begin{tabular}{lrcrr}
\hline \hline & \multicolumn{2}{c}{$[6]-[9](\mathrm{mag})$} & \multicolumn{2}{c}{ SiC/Continuum } \\
\cline { 2 - 3 } Source & \multicolumn{1}{c}{ Revised } & Original & Revised & Original \\
\hline WZ Cas & $-0.009 \pm 0.002$ & $0.029 \pm 0.002$ & $-0.021 \pm 0.003$ & $0.022 \pm 0.002$ \\
VX And & $0.155 \pm 0.003$ & $0.123 \pm 0.003$ & $0.146 \pm 0.003$ & $0.182 \pm 0.001$ \\
HV Cas & $0.339 \pm 0.002$ & $0.353 \pm 0.002$ & $0.206 \pm 0.001$ & $0.226 \pm 0.001$ \\
R Scl & $0.266 \pm 0.002$ & $0.273 \pm 0.002$ & $0.192 \pm 0.001$ & $0.205 \pm 0.001$ \\
\hline
\end{tabular}

(This table is available in its entirety in machine-readable form.)

Table 6

Revised Feature Strengths-IRS Sample

\begin{tabular}{lcccr}
\hline \hline & \multicolumn{2}{c}{$[6]-[9]$} & & \multicolumn{2}{c}{ SiC/Cont. } \\
\cline { 2 - 3 } Source & Revised & Original & Revised & Original \\
\hline MSX SMC 33 & $0.501 \pm 0.007$ & $0.514 \pm 0.009$ & $0.036 \pm 0.002$ & $0.040 \pm 0.002$ \\
MSX SMC 36 & $0.733 \pm 0.005$ & $0.774 \pm 0.005$ & $0.081 \pm 0.003$ & $0.088 \pm 0.004$ \\
MSX SMC 44 & $0.502 \pm 0.011$ & $0.517 \pm 0.006$ & $0.025 \pm 0.005$ & $0.024 \pm 0.004$ \\
MSX SMC 54 & $0.720 \pm 0.002$ & $0.756 \pm 0.004$ & $0.175 \pm 0.004$ & $0.179 \pm 0.003$ \\
\hline
\end{tabular}

(This table is available in its entirety in machine-readable form.)

small wavelength ranges. As noted in the main text, residuals from atmospheric ozone precludes the use of the same wavelength ranges that Sloan et al. (2016) used for their feature extraction for two of the four wavelengths. Specifically, the $9 \mu \mathrm{m}$ end of the [6]-[9] color has to be slightly bluer, and the short-wavelength end of the SiC feature has to be somewhat redder than the original set.

To determine the new locations, the ozone in the SOFIA data needs to be avoided, along with the $\mathrm{SiC}$ feature. We also ensure that the IRS data have enough data points to be valid, since those spectra are not as oversampled as the SOFIA data are. The SWS data are at higher spectral resolution, and thus this is not an issue for them (the smallest number of data points in a given wavelength range is 71). This changes the color from [6.4]-[9.3] to [6.4]-[9.1]. The blue end of the $\mathrm{SiC}$ feature changes from $\sim 9.8$ to $\sim 10.18 \mu \mathrm{m}$. The [6.4] band and the red end of the $\mathrm{SiC}$ feature remain unchanged. Table 4 gives the original and new wavelength ranges.

The feature strengths for the SWS and IRS samples based on the new wavelength ranges are given in Tables 5 and 6 , respectively. The values from the previous extractions (Sloan et al. 2016) are also given for comparison. Figure 12 shows the [6]-[9] versus $\mathrm{SiC} /$ Continuum for the original ranges (top panel) and for the new ranges (bottom panel). It is evident that the two plots are qualitatively the same, even though some data points have shifted slightly. We conclude that the new ranges provide reliable results for the analysis in the main part of this work.

There are a few trends that should be noted. The [6]-[9] colors from the new range are slightly bluer than the original colors, and the magnitude of the difference is a function of the color. The strength of the $\mathrm{SiC} /$ Continuum is slightly smaller with the new ranges, although this difference is not nearly as tight a function of the strength as the color difference is. Figure 13 shows the differences as functions of the original values.

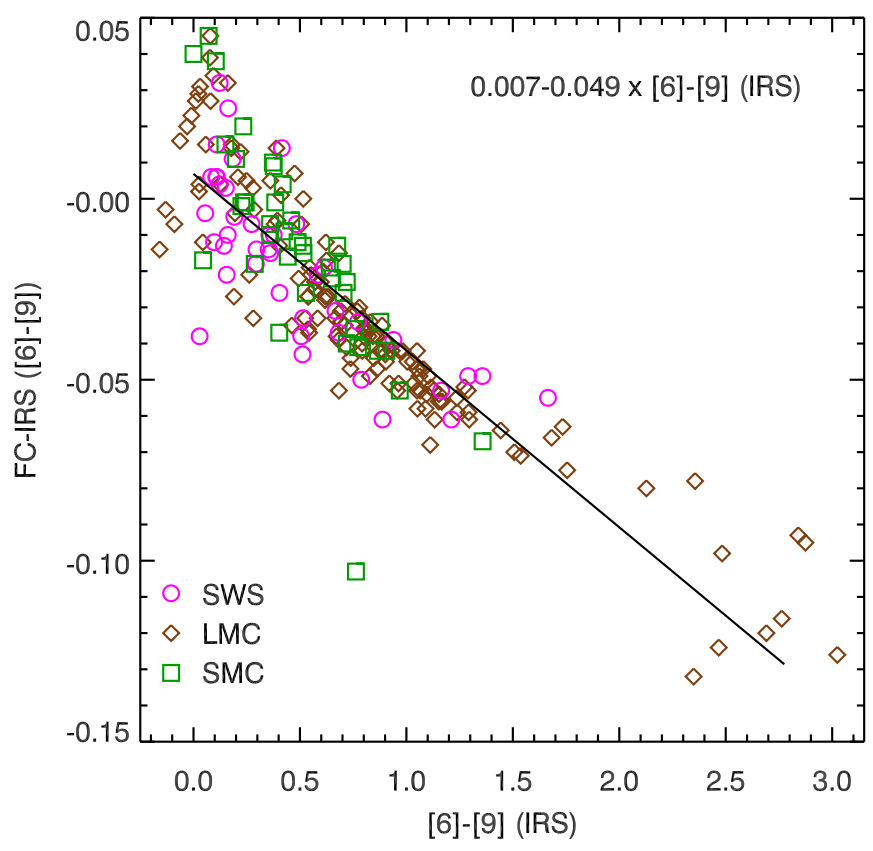

Figure 13. Difference between the [6]-[9] color from the FORCAST wavelength ranges and that from the IRS ranges as a function of the IRSderived color. Symbols are the same as those in Figure 12. The solid line is a linear fit to the data, and its parameters are given in the upper right as well as in Table 7.

We fit a line to the difference in color as a function of color for (a) the SWS data, (b) the LMC IRS data, (c) the SMC IRS data, and (d) the full SWS + IRS data set, the results of which are given in Table 7 . These can be used to adjust the results for other data sets whose features were extracted using the original wavelength ranges. 
Table 7

Linear Fits

\begin{tabular}{lcr}
\hline \hline Sample & FORCAST - IRS [6]-[9] & $N_{\text {stars }}$ \\
\hline SWS & $\Delta=0.003-0.046 \times($ IRS [6]-[9]) & 42 \\
LMC & $\Delta=0.006-0.047 \times($ IRS [6]-[9]) & 144 \\
SMC & $\Delta=0.025-0.078 \times($ IRS [6]-[9]) & 40 \\
All & $\Delta=0.007-0.049 \times($ IRS [6]-[9]) & 226 \\
\hline
\end{tabular}

\section{ORCID iDs}

Kathleen E. Kraemer (1) https://orcid.org/0000-00022626-7155

G. C. Sloan (1) https://orcid.org/0000-0003-4520-1044

Luke D. Keller (10) https://orcid.org/0000-0003-1046-512X

Iain McDonald (ํ) https://orcid.org/0000-0003-0356-0655

Albert A. Zijlstra (1) https://orcid.org/0000-0002-3171-5469

Martin A. T. Groenewegen (1) https://orcid.org/0000-00032723-6075

\section{References}

Alksnis, A., Balklavs, A., Dzervitis, U., et al. 2001, BaltA, 10, 1

Aoki, W., Tsuji, T., \& Ohnaka, K. 1998, A\&A, 340, 222

Aoki, W., Tsuji, T., \& Ohnaka, K. 1999, A\&A, 350, 945

Bladh, S., Eriksson, K., Marigo, P., Liljegren, S., \& Aringer, B. 2019, A\&A, 623, A119

Boyer, M. L., Srinivasan, S., van Loon, J. T., et al. 2011, AJ, 142, 103

Croat, T. K., Stadermann, F. J., \& Bernatowicz, T. J. 2005, ApJ, 631, 976

de Graauw, T., Haser, L. N., Beintema, D. A., et al. 1996, A\&A, 315, L49

Dell'Agli, F., García-Hernández, D. A., Schneider, R., et al. 2017, MNRAS, 467, 4431

Dell'Agli, F., Valiante, R., Kamath, D., Ventura, P., \& García-Hernández, D. A. 2019, MNRAS, 486, 4738

Díaz-Luis, J. J., Alcolea, J., Bujarrabal, V., et al. 2019, A\&A, 629, A94

Eriksson, K., Nowotny, W., Höfner, S., Aringer, B., \& Wachter, A. 2014, A\&A, 566, A95

Ferrarotti, A. S., \& Gail, H. P. 2002, A\&A, 382, 256

Fraser, O. J., Hawley, S. L., Cook, K. H., \& Keller, S. C. 2005, AJ, 129, 768

Glasby, J. S. 1968, Variable Stars (London: Constable)

Goebel, J. H., Bregman, J. D., Goorvitch, D., et al. 1980, ApJ, 235, 104

Goebel, J. H., Bregman, J. D., Witteborn, F. C., Taylor, B. J., \& Willner, S. P. 1981, ApJ, 246, 455

Groenewegen, M. A. T., \& Sloan, G. C. 2018, A\&A, 609, A114

Groenewegen, M. A. T., Wood, P. R., Sloan, G. C., et al. 2007, MNRAS, 376,313

Habing, H. J. 1996, A\&ARv, 7, 97

Herter, T. L., Adams, J. D., Buizer, J. M. D., et al. 2012, ApJL, 749, L18

Hoffmeister, C., Richter, G., \& Wenzel, W. 1984, Veraenderliche Sterne (Berlin: Springer)

Höfner, S., \& Olofsson, H. 2018, A\&ARv, 26, 1

Houck, J. R., Roellig, T. L., van Cleve, J., et al. 2004, ApJS, 154, 18

Jones, T. J., Bryja, C. O., Gehrz, R. D., et al. 1990, ApJS, 74, 785

Jones, T. W., Ney, E. P., \& Stein, W. A. 1981, ApJ, 250, 324

Jørgensen, U. G., Hron, J., \& Loidl, R. 2000, A\&A, 356, 253

Jura, M. 1983, ApJ, 267, 647

Jura, M. 1986, ApJ, 303, 327

Keller, L., Deen, C. P., Jaffe, D. T., et al. 2010, Proc. SPIE, 7735, 77356N

Kessler, M. F., Steinz, J. A., Anderegg, M. E., et al. 1996, A\&A, 315, L27

Kholopov, P. N., Samus, N. N., Durlevich, O. V., et al. 1992, BICDS, 40, 15

Kozasa, T., Dorschner, J., Henning, T., \& Stognienko, R. 1996, A\&A, 307, 551

Kraemer, K. E., Sloan, G. C., Price, S. D., \& Walker, H. J. 2002, ApJS, 140,389

Lagadec, E., \& Zijlstra, A. A. 2008, MNRAS, 390, L59

Lagadec, E., Zijlstra, A. A., Sloan, G. C., et al. 2007, MNRAS, 376, 1270

Leavitt, H. S., \& Pickering, E. C. 1912, HarCi, 173, 1

Leisenring, J. M., Kemper, F., \& Sloan, G. C. 2008, ApJ, 681, 1557

Lorenz-Martins, S., de Araújo, F. X., Codina Land aberry, S. J., de Almeida, W. G., \& de Nader, R. V. 2001, A\&A, 367, 189

Martin, P. G., \& Rogers, C. 1987, ApJ, 322, 374

Massalkhi, S., Agúndez, M., \& Cernicharo, J. 2019, A\&A, 628, A62

Matsuura, M., Barlow, M. J., Zijlstra, A. A., et al. 2009, MNRAS, 396, 918
Matsuura, M., Wood, P. R., Sloan, G. C., et al. 2006, MNRAS, 371, 415

Matsuura, M., Zijlstra, A. A., Bernard-Salas, J., et al. 2007, MNRAS, 382,1889

Matsuura, M., Zijlstra, A. A., van Loon, J. T., et al. 2002, ApJL, 580, L133

Matsuura, M., Zijlstra, A. A., van Loon, J. T., et al. 2005, A\&A, 434, 691

Mattei, J. A., Foster, G., Hurwitz, L. A., et al. 1997, in ESA Special Publication 402, Hipparcos-Venice '97, ed. R. M. Bonnet (Noordwijk: ESA), 269

Mattsson, L., Wahlin, R., Höfner, S., \& Eriksson, K. 2008, A\&A, 484, L5

McCabe, E. M. 1982, MNRAS, 200, 71

McDonald, I., De Beck, E., Zijlstra, A. A., \& Lagadec, E. 2018, MNRAS, 481, 4984

McDonald, I., \& Trabucchi, M. 2019, MNRAS, 484, 4678

McDonald, I., Uttenthaler, S., Zijlstra, A. A., Richards, A. M. S., \& Lagadec, E. 2019, MNRAS, 491, 1174

Meixner, M., Gordon, K. D., Indebetouw, R., et al. 2006, AJ, 132, 2268

Micelotta, E. R., Matsuura, M., \& Sarangi, A. 2018, SSRv, 214, 53

Nakashima, J.-I., Jiang, B. W., Deguchi, S., Sadakane, K., \& Nakada, Y. 2000 PASJ, 52, 275

Nanni, A., Groenewegen, M. A. T., Aringer, B., et al. 2019, MNRAS, 487, 502

Nanni, A., Marigo, P., Groenewegen, M. A. T., et al. 2017, MmSAI, 88, 393

Neugebauer, G., Habing, H. J., van Duinen, R., et al. 1984, ApJL, 278, L1

Olnon, F. M., Raimond, E., Neugebauer, G., et al. 1986, A\&AS, 65, 607

Payne-Gaposchkin, C., \& Gaposchkin, S. 1938, Variable Stars (Cambridge, MA: The Observatory), 5

Piatti, A. E. 2012, MNRAS, 422, 1109

Piatti, A. E., \& Geisler, D. 2013, AJ, 145, 17

Rouleau, F., \& Martin, P. G. 1991, ApJ, 377, 526

Rubele, S., Girardi, L., Kerber, L., et al. 2015, MNRAS, 449, 639

Samus, N. N., Kazarovets, E. V., Durlevich, O. V., Kireeva, N. N., \& Pastukhova, E. N. 2017, ARep, 61, 80

Sloan, G. C. 2017, in IAU Symp. 323, Planetary Nebulae: Multi-wavelength Probes of Stellar and Galactic Evolution, ed. X. Liu, L. Stanghellini, \& A. Karakas (Cambridge: Cambridge Univ. Press), 121

Sloan, G. C., Kraemer, K. E., Goebel, J. H., \& Price, S. D. 2003a, ApJ, 594,483

Sloan, G. C., Kraemer, K. E., Matsuura, M., et al. 2006, ApJ, 645, 1118

Sloan, G. C., Kraemer, K. E., McDonald, I., et al. 2016, ApJ, 826, 44

Sloan, G. C., Kraemer, K. E., McDonald, I., \& Zijlstra, A. A. 2019, in IAU Symp. 343, Why Galaxies Care About AGB Stars: A Continuing Challenge through Cosmic Time, ed. F. Kerschbaum, M. A. T. Groenewegen, \& H. Olofsson (Cambridge: Cambridge Univ. Press)

Sloan, G. C., Kraemer, K. E., Price, S. D., \& Shipman, R. F. 2003b, ApJS, 147,379

Sloan, G. C., Kraemer, K. E., Wood, P. R., et al. 2008, ApJ, 686, 1056

Sloan, G. C., Lagadec, E., Kraemer, K. E., et al. 2015, in ASP Conf. Ser. 497, Why Galaxies Care about AGB Stars III: A Closer Look in Space and Time, ed. F. Kerschbaum, R. F. Wing, \& J. Hron (San Francisco, CA: ASP), 429

Sloan, G. C., Levan, P. D., \& Little-Marenin, I. R. 1996, ApJ, 463, 310

Sloan, G. C., Little-Marenin, I. R., \& Price, S. D. 1998, AJ, 115, 809

Sloan, G. C., Matsuura, M., Lagadec, E., et al. 2012, ApJ, 752, 140

Soszyński, I., Udalski, A., Szymański, M. K., et al. 2009, AcA, 59, 239

Soszyński, I., Udalski, A., Szymański, M. K., et al. 2011, AcA, 61, 217

Soszyński, I., Wood, P. R., \& Udalski, A. 2013, ApJ, 779, 167

Speck, A. K., Cami, J., Markwick-Kemper, C., et al. 2006, ApJ, 650, 892

Udalski, A., Szymanski, M., Kaluzny, J., Kubiak, M., \& Mateo, M. 1992, AcA, 42,253

Uttenthaler, S., McDonald, I., Bernhard, K., Cristallo, S., \& Gobrecht, D. 2019 , A\&A, 622, A120

van Loon, J. T., Marshall, J. R., Cohen, M., et al. 2006, A\&A, 447, 971

van Loon, J. T., Zijlstra, A. A., \& Groenewegen, M. A. T. 1999, A\&A, 346,805

Wallerstein, G., \& Knapp, G. R. 1998, ARA\&A, 36, 369

Werner, M. W., Roellig, T. L., Low, F. J., et al. 2004, ApJS, 154, 1

Whitelock, P. A., Feast, M. W., Marang, F., \& Groenewegen, M. A. T. 2006, MNRAS, 369, 751

Whitelock, P. A., Feast, M. W., van Loon, J. T., \& Zijlstra, A. A. 2003 , MNRAS, 342, 86

Wood, P. R., Alcock, C., Allsman, R. A., et al. 1999, in IAU Symp. 191, Asymptotic Giant Branch Stars, ed. T. Le Bertre, A. Lebre, \& C. Waelkens (Cambridge: Cambridge Univ. Press), 151

Wood, P. R., \& Sebo, K. M. 1996, MNRAS, 282, 958

Wright, E. L., Eisenhardt, P. R. M., Mainzer, A. K., et al. 2010, AJ, 140, 1868 Young, E. T., Becklin, E. E., Marcum, P. M., et al. 2012, ApJL, 749, L17

Zijlstra, A. A., Matsuura, M., Wood, P. R., et al. 2006, MNRAS, 370, 1961

Zubko, V., Dwek, E., \& Arendt, R. G. 2004, ApJS, 152, 211 